Supporting Information

\title{
Formation and Conformational Conversion of Flattened Partial Cone Oxygen Bridged Calix[2]arene[2]triazines
}

Qi-Qiang Wang, De-Xian Wang, Qi-Yu Zheng and Mei-Xiang Wang*

National Laboratory for Molecular Sciences, Laboratory of Chemical Biology, Institute of Chemistry, Chinese Academy of Sciences, Beijing 100080, China

mxwang@iccas.ac.cn

\section{Table of Contents}

General information

Synthetic procedures and characterization data for compounds 3-5, 7-9,

Copies of ${ }^{1} \mathrm{H}$ and ${ }^{13} \mathrm{C}$ NMR Spectra for 3-5, 7-9, 11-13 


\section{General information}

${ }^{1} \mathrm{H}$ and ${ }^{13} \mathrm{C}$ NMR spectra were recorded on a Bruker Avance 300 spectrometer. Chemical shifts are reported in ppm versus tetramethylsilane with either tetramethylsilane or the residual solvent resonance used as an internal standard. Melting points are uncorrected. Elemental analyses were performed at the Analytical Laboratory of the Institute. All chemicals were dried or purified according to standard procedures prior to use.

Synthetic procedures and characterization data for compounds 3-5, 7-9, 11-13

3: To an ice-bath cooled solution of cyanuric chloride 2 (4.06 g, $22 \mathrm{mmol})$ in THF $(60 \mathrm{~mL})$ was added dropwise a mixture of Methyl 4-(Benzyloxy)-3,5-dihydroxybenzoate $\quad \mathbf{1}^{1} \quad\left(\begin{array}{llll}2.74 & \mathrm{~g}, & 10 & \mathrm{mmol}\end{array}\right)$ and diisopropylethylamine $(3.23 \mathrm{~g}, 25 \mathrm{mmol})$ in THF $(40 \mathrm{~mL})$ during $1 \mathrm{~h}$. The reaction mixture was stirred for another $2 \mathrm{~h}$. After removal of diisopropylethylamine hydrochloride salt through filtration, the filtrate was concentrated and chromatographed on a silica gel column (200-300) with a mixture of petroleum ether and ethyl acetate as the mobile phase to give pure 3 (4.21 g, 74\%) as a white solid: mp $173-174{ }^{\circ} \mathrm{C} ;{ }^{1} \mathrm{H}$ NMR $\left(\mathrm{CDCl}_{3} / 300 \mathrm{MHz}\right)$ $\delta 7.88(\mathrm{~s}, 2 \mathrm{H}), 7.29-7.25(\mathrm{~m}, 3 \mathrm{H}), 7.15-7.11(\mathrm{~m}, 2 \mathrm{H}), 5.08(\mathrm{~s}, 2 \mathrm{H}), 3.94(\mathrm{~s}$, $3 \mathrm{H}) ;{ }^{13} \mathrm{C}$ NMR $\left(75 \mathrm{MHz} / \mathrm{CDCl}_{3}\right) \delta 173.2,170.6,164.5,146.3,144.4,135.1$, 128.7, 128.5, 127.3, 126.4, 122.7, 76.1, 52.7; IR (KBr) 1730, $1524 \mathrm{~cm}^{-1}$; MS (EI) $\mathrm{m} / z 574$ (1), 572 (2), 570 (3), $568\left(\mathrm{M}^{+}, 3\right), 91$ (100). Anal. Calcd. for $\mathrm{C}_{21} \mathrm{H}_{12} \mathrm{~N}_{6} \mathrm{O}_{5} \mathrm{Cl}_{4}: \mathrm{C}, 44.24 ; \mathrm{H}, 2.12 ; \mathrm{N}, 14.74$. Found: C, 44.56; H, 2.11; N, 14.87. 
4: At room temperature, both solutions of Methyl 4-(Benzyloxy)-3,5-dihydroxybenzoate 1 (1.37 g, $5 \mathrm{mmol})$ in acetone (250 $\mathrm{mL})$ and the trimer $3(2.85 \mathrm{~g}, 5 \mathrm{mmol})$ in acetone $(250 \mathrm{~mL})$ were added dropwise at the same time and the same rate to a solution of diisopropylethylamine $(1.61 \mathrm{~g}, 12.5 \mathrm{mmol})$ in acetone $(500 \mathrm{~mL})$. After addition of two reactants, which took about $8 \mathrm{~h}$, the resulting mixture was stirred at room temperature for another 2 days. The solvents were then removed, and the residue was chromatographed on a silica gel column (200-300) with a mixture of petroleum ether and dichloromethane as the mobile phase to give pure $4(1.64 \mathrm{~g}, 43 \%)$ as a white solid, recrystallization from a mixture of $n$-hexane and dichloromethane gave colorless prisms: $\mathrm{mp}$ 230-232 ${ }^{\circ} \mathrm{C} ;{ }^{1} \mathrm{H}$ NMR $\left(\mathrm{CDCl}_{3} / 300 \mathrm{MHz}\right) \delta 7.62$ (s, 4H), 7.30-7.19 (m, 6H), 7.08-7.04 (m, 4H), $4.89(\mathrm{~s}, 4 \mathrm{H}), 3.88(\mathrm{~s}, 6 \mathrm{H}) ;{ }^{13} \mathrm{C} \mathrm{NMR}\left(75 \mathrm{MHz} / \mathrm{CDCl}_{3}\right) \delta$ 174.2, 171.7, 164.6, 146.2, 144.6, 135.1, 128.7, 128.4, 127.7, 126.1, 122.1, 76.1, 52.6; IR (KBr) 1727, 1603, $1550 \mathrm{~cm}^{-1}$; MS (MALDI-TOF) $\mathrm{m} / \mathrm{z} 771.2$ $\left(\mathrm{M}^{+}+1,18\right), 772.2$ (10), 773.2 (15), 774.2 (6), $793.2\left(\mathrm{M}^{+}+\mathrm{Na}, 100\right), 794.2$ (40), 795.2 (65), 796.2 (23), 797.2 (15). Anal. Calcd. for $\mathrm{C}_{36} \mathrm{H}_{24} \mathrm{~N}_{6} \mathrm{O}_{10} \mathrm{Cl}_{2}$ : C, 56.04; H, 3.14; N, 10.89. Found: C, 56.20; H, 3.09; N, 10.91.

5: Anhydrous $\mathrm{AlCl}_{3}(2.0 \mathrm{~g}, 15 \mathrm{mmol})$ was added to dry toluene $(100 \mathrm{~mL})$ and stirred for 5 minutes. 4 (386 $\mathrm{mg}, 0.5 \mathrm{mmol}$ ) was added to the suspension and stirred at room temperature for $12 \mathrm{~h}$. The reaction mixture was poured into ice-water mixture $(100 \mathrm{~mL})$, and acidified with excess $10 \% \mathrm{HCl}$ to neutralize the unreacted $\mathrm{AlCl}_{3}$. The organic phase was separated, and the water phase was extracted with EtOAc $(2 \times 150 \mathrm{~mL})$. The combined organic extracts were dired over $\mathrm{Na}_{2} \mathrm{SO}_{4}$, evaporated under vacuum and chromatographed on a silica gel column (100-200) with a mixture of 
petroleum ether and ethyl acetate as the mobile phase to give 5 (263 mg, $75 \%$ ) as a white solid: $\mathrm{mp}>300{ }^{\circ} \mathrm{C} ;{ }^{1} \mathrm{H}$ NMR (DMSO- $\left.d_{6} / 300 \mathrm{MHz}\right) \delta 10.87$ (s, 2H, OH), 8.39 (d, J=8.2Hz, 4H), $7.50(\mathrm{~s}, 4 \mathrm{H}), 7.45$ (d, J=8.2Hz, 4H), $3.74(\mathrm{~s}, 6 \mathrm{H}), 2.45(\mathrm{~s}, 6 \mathrm{H}) ;{ }^{13} \mathrm{C}$ NMR $\left(75 \mathrm{MHz} / \mathrm{DMSO}-d_{6}\right) \delta 175.2,172.1$, 164.6, 147.0, 143.9, 140.1, 131.5, 129.7, 128.7, 121.7, 119.2, 51.9, 21.2; IR (KBr) 3604, 3459, 1703, 1577, $1568 \mathrm{~cm}^{-1}$; MS (ESI-neg) m/z 701.1 (M-1). Anal. Calcd. for $\mathrm{C}_{36} \mathrm{H}_{26} \mathrm{~N}_{6} \mathrm{O}_{10}$ : C, 61.54; H, 3.73; N, 11.96. Found: C, 61.31; $\mathrm{H}, 3.96 ; \mathrm{N}, 11.95$.

7: To a solution of 2, 4-dichloro-6-p-tolyl-[1, 3, 5]-triazine 6 (3.84 g, 16 $\mathrm{mmol})$ in THF $(60 \mathrm{~mL})$ was added dropwise a mixture of Methyl 4-(Benzyloxy)-3,5-dihydroxybenzoate $\quad \mathbf{1}(2.19 \mathrm{~g}, \quad 8 \quad \mathrm{mmol})$ and diisopropylethylamine (2.58 g, $20 \mathrm{mmol})$ in THF (40 mL) during $1.5 \mathrm{~h}$. The reaction mixture was stirred at room temperature for another $40 \mathrm{~h}$. After removal of diisopropylethylamine hydrochloride salt through filtration, the filtrate was concentrated and chromatographed on a silica gel column (200-300) with a mixture of petroleum ether and dichloromethane as the mobile phase to give pure 7 (4.07 g, $75 \%$ ) as a white solid: $\mathrm{mp} 167-168{ }^{\circ} \mathrm{C}$; ${ }^{1} \mathrm{H}$ NMR $\left(\mathrm{CDCl}_{3} / 300 \mathrm{MHz}\right) \delta 8.20(\mathrm{~d}, J=8.3 \mathrm{~Hz}, 4 \mathrm{H}), 7.93(\mathrm{~s}, 2 \mathrm{H}), 7.21$ (d, $J=8.1 \mathrm{~Hz}, 4 \mathrm{H}), 7.08-7.01(\mathrm{~m}, 5 \mathrm{H}), 5.10(\mathrm{~s}, 2 \mathrm{H}), 3.93(\mathrm{~s}, 3 \mathrm{H}), 2.41(\mathrm{~s}, 6 \mathrm{H})$; ${ }^{13} \mathrm{C}$ NMR $\left(75 \mathrm{MHz} / \mathrm{CDCl}_{3}\right) \delta 175.4,172.8,170.9,165.1,147.1,145.2,145.0$, 135.6, 130.7, 129.7, 129.62, 128.2, 128.16, 127.1, 125.8, 122.6, 75.7, 52.6, 21.8; IR (KBr) 1732, $1546 \mathrm{~cm}^{-1}$; MS (ESI) $\mathrm{m} / \mathrm{z} 681.3\left(\mathrm{M}^{+}+1,100\right), 682.3$ (41), 683.3 (58), 703.2 ( $\left.\mathrm{M}^{+}+\mathrm{Na}, 29\right), 704.2$ (25), 705.2 (18), 706.2 (6). Anal. Calcd. for $\mathrm{C}_{35} \mathrm{H}_{26} \mathrm{~N}_{6} \mathrm{O}_{5} \mathrm{Cl}_{2}: \mathrm{C}, 61.68 ; \mathrm{H}, 3.85 ; \mathrm{N}, 12.33$. Found: $\mathrm{C}, 61.77 ; \mathrm{H}$, 3.94; N, 12.13 . 
8: Both solutions of Methyl 4-(Benzyloxy)-3,5-dihydroxybenzoate 1 (274 $\mathrm{mg}, 1 \mathrm{mmol})$ in acetone, THF, dioxane or $\mathrm{MeCN}(50 \mathrm{~mL})$ and the trimer 7 (681 $\mathrm{mg}, 1 \mathrm{mmol})$ in the same solvent $(50 \mathrm{~mL})$ were added dropwise at the same time and the same rate to a solution or a suspension of diisopropylethylamine (323 mg, $2.5 \mathrm{mmol}), \mathrm{Et}_{3} \mathrm{~N}(253 \mathrm{mg}, 2.5 \mathrm{mmol}$ ), finely ground $\mathrm{K}_{2} \mathrm{CO}_{3}(173 \mathrm{mg}, 1.25 \mathrm{mmol})$ or $\mathrm{Cs}_{2} \mathrm{CO}_{3}(408 \mathrm{mg}, 1.25 \mathrm{mmol})$ in the same solvent $(100 \mathrm{~mL})$ at reflux for about $4 \mathrm{~h}$ (when without dropping, the reactants were mixed directly and then refluxed). The resulting mixture was refluxed for a period of time (Scheme 2 and Table 1). After reaction, for entries $8-10$, the product precipitated as white solids, filtration and wash with water gave pure product 8a. For entries 1, 2, 5-7, filtration gave a majority of pure product $\mathbf{8 a}$, the filtrate was concentrated and chromatographed on a silica gel column (200-300) with a mixture of petroleum ether and dichloromethane as the mobile phase to give an another part of $\mathbf{8 a}$ and $\mathbf{8 b}$ as white solids. 8a, recrystallization from a mixture of $n$-hexane and dichloromethane gave colorless thin platelets: $\mathrm{mp}>300{ }^{\circ} \mathrm{C} ;{ }^{1} \mathrm{H}$ NMR $\left(\mathrm{CDCl}_{3} / 300 \mathrm{MHz}\right) \delta 8.42(\mathrm{~d}, J=8.3 \mathrm{~Hz}, 4 \mathrm{H}), 7.64(\mathrm{~s}, 4 \mathrm{H}), 7.35(\mathrm{~d}$, $J=8.0 \mathrm{~Hz}, 4 \mathrm{H}), 6.99-6.95$ (m, 10H), 4.90 (s, 4H), 3.87 (s, 6H), 2.49 (s, 6H); ${ }^{13} \mathrm{C}$ NMR $\left(75 \mathrm{MHz} / \mathrm{CDCl}_{3}\right) \delta 176.8,172.3,165.0,147.1,145.0,144.3,135.7$, 131.7, 129.45, 129.4, 128.1, 128.0, 127.6, 125.6, 122.1, 75.5, 52.4, 21.8; IR (KBr) 1722, 1577, $1564 \mathrm{~cm}^{-1}$; MS (MALDI-TOF) m/z $883.4\left(\mathrm{M}^{+}+1\right), 905.4$ $\left(\mathrm{M}^{+}+\mathrm{Na}\right), 921.4\left(\mathrm{M}^{+}+\mathrm{K}\right)$. Anal. Calcd. for $\mathrm{C}_{50} \mathrm{H}_{38} \mathrm{~N}_{6} \mathrm{O}_{10}: \mathrm{C}, 68.02 ; \mathrm{H}, 4.34 ; \mathrm{N}$, 9.52. Found: $\mathrm{C}, 68.12 ; \mathrm{H}, 4.44 ; \mathrm{N}, 9.53 .8 \mathrm{~b}$, recrystallization from a mixture of acetone and dichloromethane gave colorless thin platelets: $\mathrm{mp} 296-298{ }^{\circ} \mathrm{C}$; ${ }^{1} \mathrm{H}$ NMR $\left(\mathrm{CDCl}_{3} / 300 \mathrm{MHz}\right) \delta 8.52(\mathrm{~d}, J=8.3 \mathrm{~Hz}, 4 \mathrm{H}), 7.59(\mathrm{~s}, 4 \mathrm{H}), 7.39(\mathrm{~d}$, $J=8.0 \mathrm{~Hz}, 4 \mathrm{H}), 7.18-7.14$ (m, 6H), 6.99-6.95 (m, 4H), 4.92 (s, 4H), 3.69 (s, $6 \mathrm{H}), 2.50(\mathrm{~s}, 6 \mathrm{H}) ;{ }^{13} \mathrm{C} \mathrm{NMR}\left(75 \mathrm{MHz} / \mathrm{CDCl}_{3}\right) \delta 177.1,174.0,164.4,150.0$, 
$146.0,144.9,136.3,131.4,129.7,129.68,128.2,127.8,127.0,124.7,123.1$, 74.2, 52.2, 21.9; IR (KBr) 1723, $1558 \mathrm{~cm}^{-1}$; MS (MALDI-TOF) $\mathrm{m} / z 883.5$ $\left(\mathrm{M}^{+}+1\right), 905.5\left(\mathrm{M}^{+}+\mathrm{Na}\right), 921.5\left(\mathrm{M}^{+}+\mathrm{K}\right)$. Anal. Calcd. for $\mathrm{C}_{50} \mathrm{H}_{38} \mathrm{~N}_{6} \mathrm{O}_{10}: \mathrm{C}$, 68.02; H, 4.34; N, 9.52. Found: C, 67.81; H, 4.52; N, 9.46.

11: To a solution of 2-tert-butyl-4, 6-dichloro-[1, 3, 5]-triazine $\mathbf{1 0}(2.47 \mathrm{~g}, 12$ $\mathrm{mmol}$ ) in THF (30 mL) was added dropwise a mixture of 2-(Benzyloxy)-1, 3-dihydroxy-5-t-butylbenzol 9 (1.36 g, $5 \mathrm{mmol})$ and diisopropylethylamine $(1.61 \mathrm{~g}, 12.5 \mathrm{mmol})$ in THF $(20 \mathrm{~mL})$ during $1 \mathrm{~h}$. The reaction mixture was stirred at room temperature for another $66 \mathrm{~h}$. After removal of diisopropylethylamine hydrochloride salt through filtration, the filtrate was concentrated and chromatographed on a silica gel column (200-300) with a mixture of petroleum ether and ethyl acetate as the mobile phase to give pure $11(2.27 \mathrm{~g}, 74 \%)$ as a white solid: $\mathrm{mp}$ 138-139 ${ }^{\circ} \mathrm{C}$; ${ }^{1} \mathrm{H}$ NMR $\left(\mathrm{CDCl}_{3} / 300 \mathrm{MHz}\right) \delta$ 7.20-7.16 (m, 3H), $7.14(\mathrm{~s}, 2 \mathrm{H}), 7.10-7.06(\mathrm{~m}, 2 \mathrm{H}), 4.96$ $(\mathrm{s}, 2 \mathrm{H}), 1.32(\mathrm{~s}, 9 \mathrm{H}), 1.28(\mathrm{~s}, 18 \mathrm{H}) ;{ }^{13} \mathrm{C} \mathrm{NMR}\left(75 \mathrm{MHz} / \mathrm{CDCl}_{3}\right) \delta 190.7$, $172.5,170.9,147.7,144.9,140.2,136.6,128.2,127.9,127.2,117.9,75.3$, 39.9, 34.8, 31.2, 28.5; IR (KBr) 1552, $1519 \mathrm{~cm}^{-1}$; MS (ESI) $\mathrm{m} / \mathrm{z} 611.4\left(\mathrm{M}^{+}+1\right.$, 100), 612.4 (33), 613.4 (74), 614.4 (28). Anal. Calcd. for $\mathrm{C}_{31} \mathrm{H}_{36} \mathrm{~N}_{6} \mathrm{O}_{3} \mathrm{Cl}_{2}$ : C, $60.88 ; \mathrm{H}, 5.93 ; \mathrm{N}, 13.74$. Found: C, 60.86; H, 5.95; N, 13.54 .

12: 2-(Benzyloxy)-1, 3-dihydroxy-5-t-butylbenzol 9 (1 eq.), the trimer 11 (1 eq.) and the base (1.25 eq.) were added to acetone or $\mathrm{MeCN}$, the resulting mixture was refluxed for a period of time (Scheme 3 and Table 2). After reaction, for entries $2-3$, the product precipitated as white solids, filtration and wash with water gave pure product 12a. For entries 4-5, the reaction mixture was concentrated and chromatographed on a silica gel column 
(200-300) with a mixture of petroleum ether and ethyl acetate as the mobile phase to give 12a and $\mathbf{1 2 b}$ as white solids. 12a, recrystallization from acetone gave colorless blocks: $\mathrm{mp}>300{ }^{\circ} \mathrm{C} ;{ }^{1} \mathrm{H}$ NMR $\left(\mathrm{CDCl}_{3} / 300 \mathrm{MHz}\right) \delta$ 7.08 (tt, $J=7.1, J=1.3 \mathrm{~Hz} 2 \mathrm{H}), 6.99$ (t, $J=7.1 \mathrm{~Hz}, 4 \mathrm{H}), 6.93$ (s, 4H), 6.91 (s, $4 \mathrm{H}), 4.78(\mathrm{~s}, 4 \mathrm{H}), 1.36(\mathrm{~s}, 18 \mathrm{H}), 1.17(\mathrm{~s}, 18 \mathrm{H}) ;{ }^{13} \mathrm{C} \mathrm{NMR}\left(75 \mathrm{MHz} / \mathrm{CDCl}_{3}\right) \delta$ $191.3,172.4,147.5,144.9,140.5,136.8,128.0,127.4,127.1,117.0,75.0$, 39.5, 34.5, 31.2, 28.7; IR (KBr) 1574, $1543 \mathrm{~cm}^{-1}$; MS (MALDI-TOF) $\mathrm{m} / \mathrm{z}$ $811.8\left(\mathrm{M}^{+}+1\right), 833.8\left(\mathrm{M}^{+}+\mathrm{Na}\right), 849.8\left(\mathrm{M}^{+}+\mathrm{K}\right)$. Anal. Calcd. for $\mathrm{C}_{48} \mathrm{H}_{54} \mathrm{~N}_{6} \mathrm{O}_{6}$ : C, 71.09; H, 6.71; N, 10.36. Found: C, 70.91; H, 6.72; N, 10.56. 12b, recrystallization from acetone gave colorless blocks: mp $280-282{ }^{\circ} \mathrm{C} ;{ }^{1} \mathrm{H}$ NMR $\left(\mathrm{CDCl}_{3} / 300 \mathrm{MHz}\right) \delta 7.18-7.13(\mathrm{~m}, 6 \mathrm{H}), 6.98-6.95(\mathrm{~m}, 4 \mathrm{H}), 6.86(\mathrm{~s}$, $4 \mathrm{H}), 4.75(\mathrm{~s}, 4 \mathrm{H}), 1.51(\mathrm{~s}, 18 \mathrm{H}), 1.00(\mathrm{~s}, 18 \mathrm{H}) ;{ }^{13} \mathrm{C} \mathrm{NMR}\left(75 \mathrm{MHz} / \mathrm{CDCl}_{3}\right) \delta$ $191.7,173.7,146.8,145.9,143.5,137.6,128.0,127.6,127.2,118.3,74.3$, 39.8, 34.2, 30.8, 28.9; IR (KBr) 1564, $1534 \mathrm{~cm}^{-1}$; MS (MALDI-TOF) $\mathrm{m} / \mathrm{z}$ $811.8\left(\mathrm{M}^{+}+1\right), 833.7\left(\mathrm{M}^{+}+\mathrm{Na}\right), 849.7\left(\mathrm{M}^{+}+\mathrm{K}\right)$. Anal. Calcd. for $\mathrm{C}_{48} \mathrm{H}_{54} \mathrm{~N}_{6} \mathrm{O}_{6}$ : C, 71.09; H, 6.71; N, 10.36. Found: C, 71.33; H, 6.75; N, 10.48.

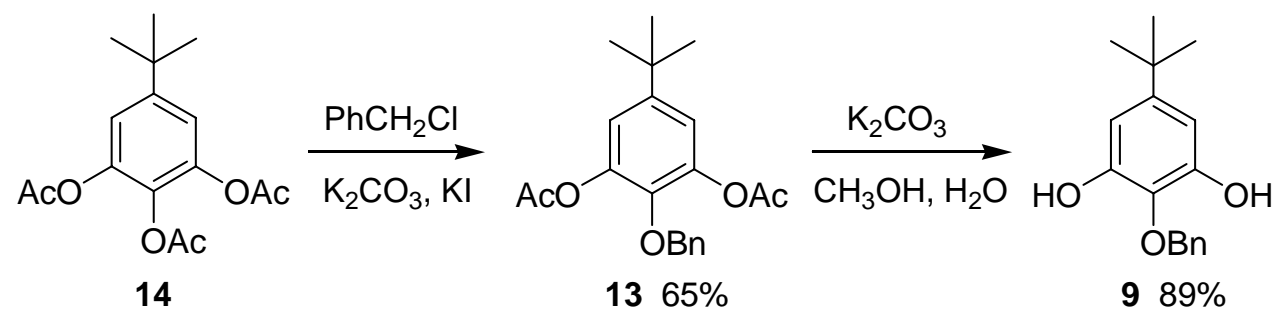

2-(Benzyloxy)-1, $\quad 3$-diacetoxy-5-t-butylbenzol $\quad \mathbf{1 3}^{1}: \quad 1, \quad 2$, 3-triacetoxy-5-t-butylbenzol $14^{2}(4.00 \mathrm{~g}, 13 \mathrm{mmol})$, benzyl chloride $(3.0 \mathrm{~mL}$, $26 \mathrm{mmol}), \mathrm{K}_{2} \mathrm{CO}_{3}(5.44 \mathrm{~g}, 39.4 \mathrm{mmol}), \mathrm{KI}(332 \mathrm{mg}, 2.0 \mathrm{mmol})$ were added to acetone $(80 \mathrm{~mL})$, the resulting mixture was refluxed for $66 \mathrm{~h}$. After reaction, the mixture was concentrated and the residue was extracted with 
water $(40 \mathrm{~mL})$ and diethyl ether $(2 \times 60 \mathrm{~mL})$. The combined organic phase was dried over $\mathrm{Na}_{2} \mathrm{SO}_{4}$, and chromatographed on a silica gel column (200-300) with a mixture of petroleum ether and ethyl acetate as the mobile phase to give 13 (3.02 g, 65\%) as a white solid: mp 75-76 ${ }^{\circ} \mathrm{C}$; ${ }^{1} \mathrm{H}$ NMR $\left(\mathrm{CDCl}_{3} / 300 \mathrm{MHz}\right) \delta$ 7.37-7.25 (m, 5H), $6.97(\mathrm{~s}, 2 \mathrm{H}), 4.94(\mathrm{~s}, 2 \mathrm{H}), 2.17(\mathrm{~s}$, $6 \mathrm{H}), 1.29$ (s, 9H); ${ }^{13} \mathrm{C} \mathrm{NMR}\left(75 \mathrm{MHz} / \mathrm{CDCl}_{3}\right) \delta 168.8,147.4,144.0,140.7$, 137.4, 128.4, 128.0, 127.6, 118.0, 75.4, 34.6, 31.2, 20.7; IR (KBr) 1773, $1747 \mathrm{~cm}^{-1}$; MS (EI) m/z 356 (M+1, 1), 314 (10), 272 (8), 181 (8), 91 (100). Anal. Calcd. for $\mathrm{C}_{21} \mathrm{H}_{24} \mathrm{O}_{5}$ : C, 70.77; H, 6.79. Found: C, 70.88; H, 6.90.

2-(Benzyloxy)-1, 3-dihydroxy-5-t-butylbenzol 9: To a solution of 2-(Benzyloxy)-1, 3-diacetoxy-5-t-butylbenzol 13 (3.0 g, $8.4 \mathrm{mmol})$ in $\mathrm{CH}_{3} \mathrm{OH}(50 \mathrm{~mL})$ was added a solution of $\mathrm{K}_{2} \mathrm{CO}_{3}(7.65 \mathrm{~g}, 55.4 \mathrm{mmol})$ in water $(20 \mathrm{~mL})$. The resulting mixture was stirred for 20 minutes at room temperature, and then concentrated under vacuum to remove $\mathrm{CH}_{3} \mathrm{OH}$. The residue was acidified with excess $10 \% \mathrm{HCl}$ to $\mathrm{pH}=2$. The acidified aqueous solution was extracted with EtOAc $(2 \times 60 \mathrm{~mL})$. The combined EtOAc extracts were washed with brine $(40 \mathrm{~mL})$, dired over $\mathrm{Na}_{2} \mathrm{SO}_{4}$ and evaporated in vacuo. Recrystallization from $n$-hexane gave 9 (2.02 g, 89\%) as an orange red solid: $\mathrm{mp} 71-72{ }^{\circ} \mathrm{C} ;{ }^{1} \mathrm{H}$ NMR $\left(\mathrm{CDCl}_{3} / 300 \mathrm{MHz}\right) \delta$ 7.44-7.38 (m, 5H), $6.52(\mathrm{~s}, 2 \mathrm{H}), 5.20(\mathrm{~s}, \mathrm{br}, 2 \mathrm{H}), 4.98(\mathrm{~s}, 2 \mathrm{H}), 1.25(\mathrm{~s}, 9 \mathrm{H}) ;{ }^{13} \mathrm{C}$ NMR $\left(75 \mathrm{MHz} / \mathrm{CDCl}_{3}\right) \delta 148.8,148.4,136.8,131.3,129.0,128.9,128.4,105.4$, 76.0, 34.5, 31.3; IR (KBr) 3543, 3494, 3376, $1597 \mathrm{~cm}^{-1}$; MS (EI) m/z 273 (3), $272\left(\mathrm{M}^{+}\right.$, 13), 91 (100). Exact mass for $\mathrm{C}_{17} \mathrm{H}_{20} \mathrm{O}_{3}$ : 272.1412. Found: 272.1415 . 
Reference

(1). Pearson, A. J. Bruhn, P. R. J. Org. Chem. 1991, 56, 7092

(2). Blatchly, J. M. Green, R. J. S. McOmie, J. F. W. J. Chem. Soc., Perkin

Trans. 1. 1972, 2286

Copies of ${ }^{1} \mathrm{H}$ and ${ }^{13} \mathrm{C}$ NMR Spectra for $3-5,7-9,11-13$ 
${ }^{1} \mathrm{H}$ NMR of 3

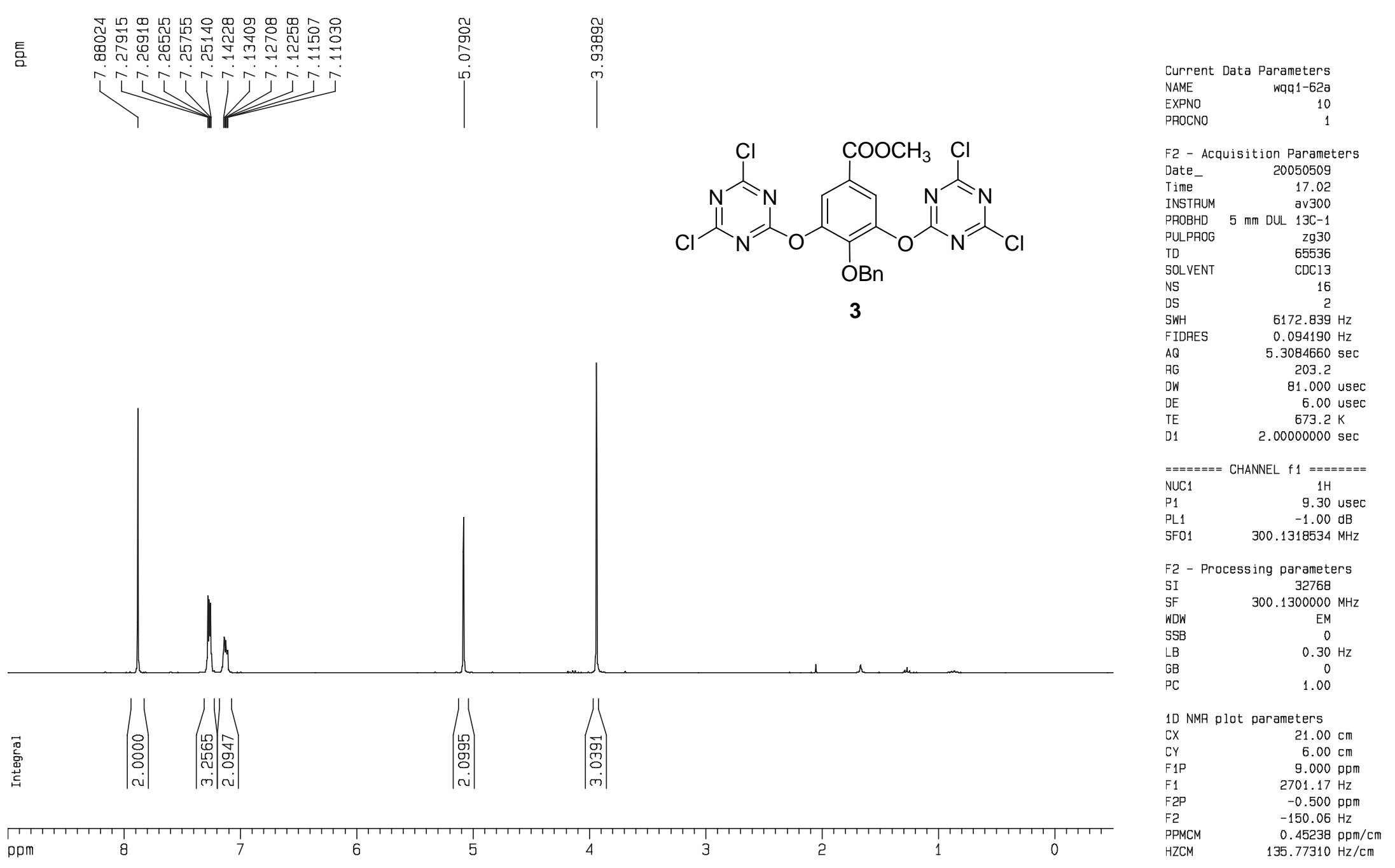


${ }^{13} \mathrm{C} \mathrm{NMR}$ of 3

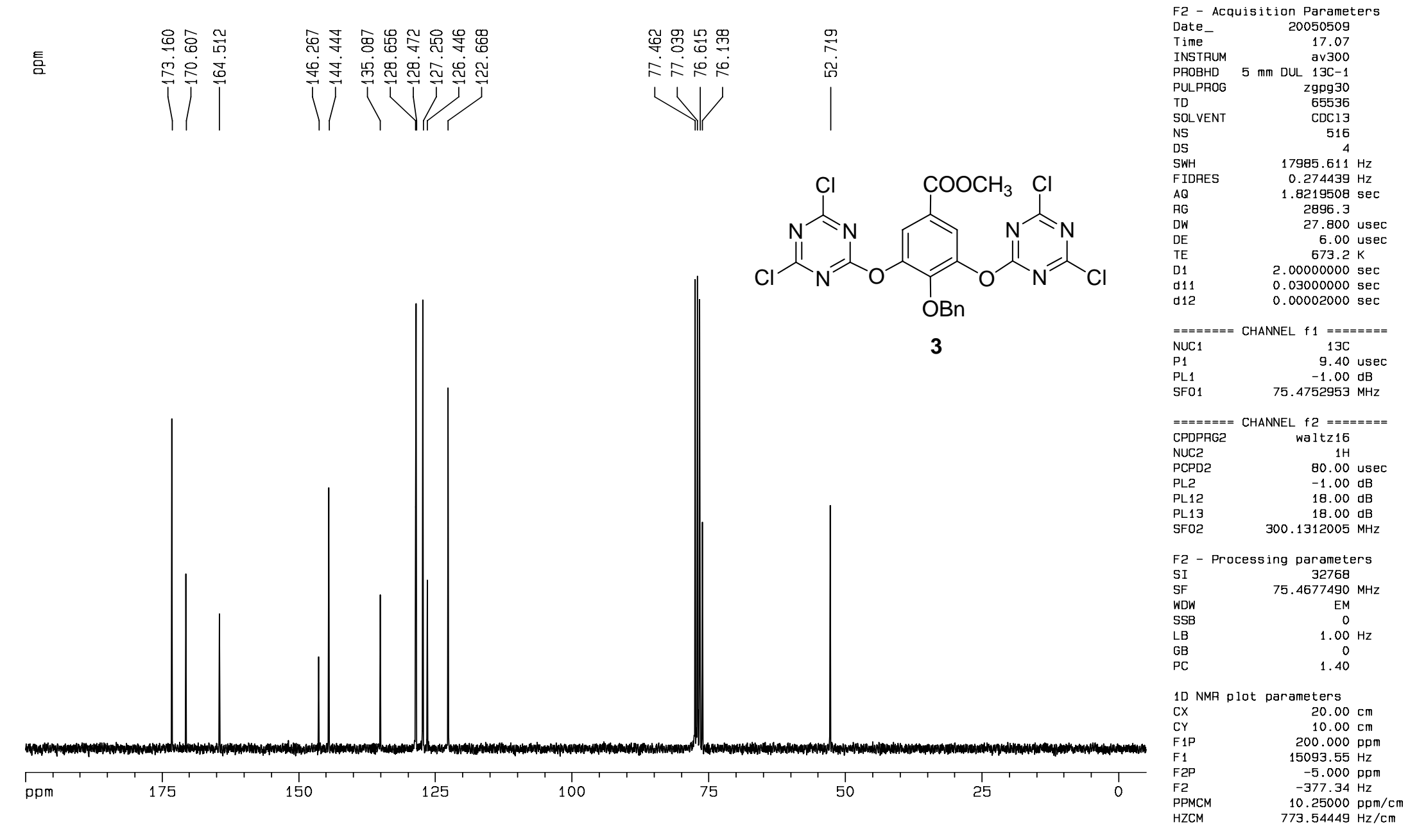


${ }^{1} \mathrm{HNMR}$ of $\mathbf{4}$

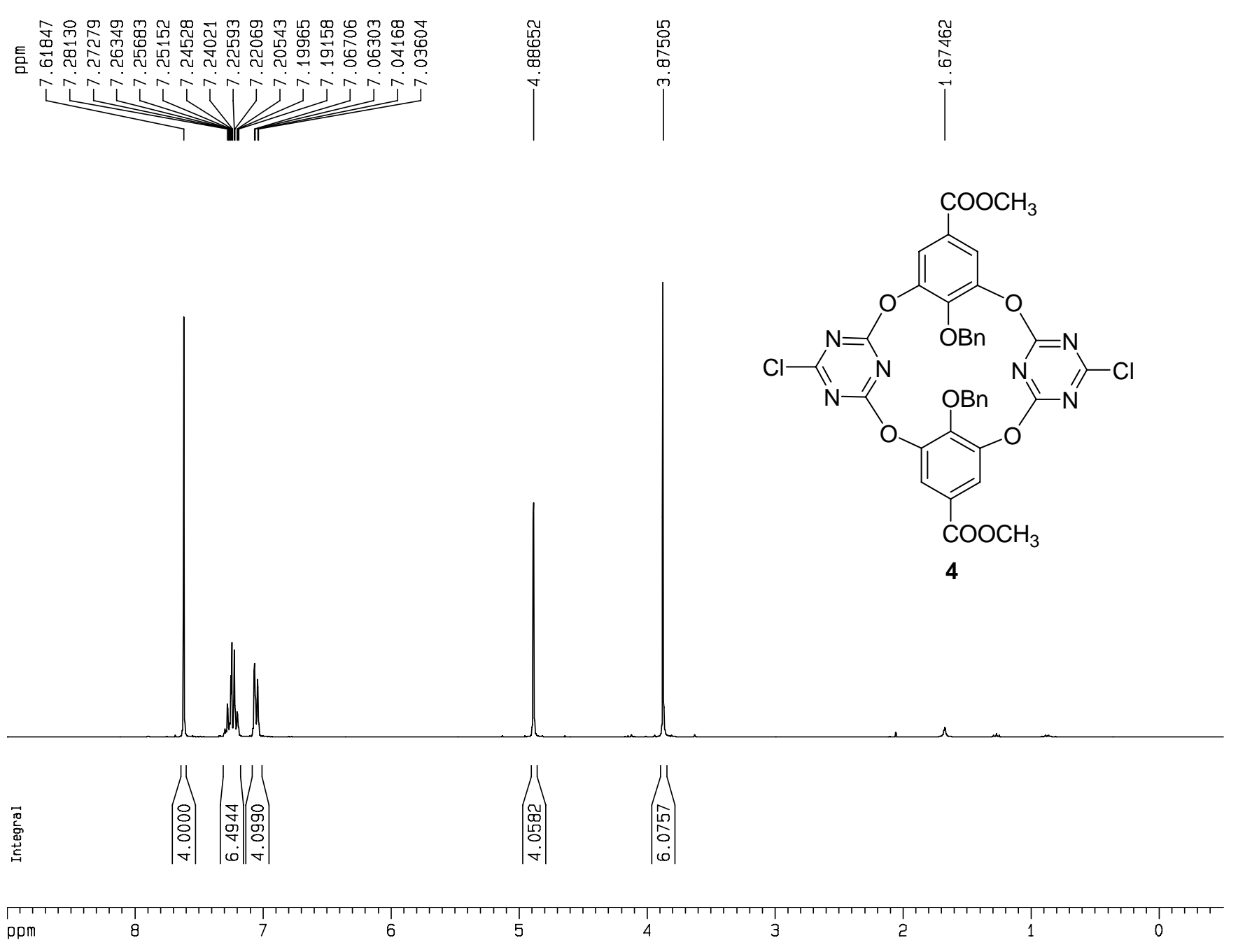

Current Data Parameters

$\begin{array}{lr}\text { NAME } & \text { WqQ1-63b } \\ \text { EXPNO } & 10\end{array}$

F2 - Acquisition Parameters

Date $\quad 20050516$

INSTRUM $\quad 0.38$

PROBHD $5 \mathrm{~mm}$ DUL $13 \mathrm{C}-1$

$\begin{array}{lr}\text { PULPROG } & 2930 \\ \text { ID } & 65536\end{array}$

$\begin{array}{ll}\text { TD } & 65536 \\ \text { SOLVENT } & \text { CDC13 }\end{array}$

$\begin{array}{lr}\text { NS } & 13 \\ \text { DS } & 2\end{array}$

SWH $\quad 5172.839 \mathrm{~Hz}$

$0.094190 \mathrm{~Hz}$
$\mathrm{FIDRES}$

AQ $\quad 5.3084660 \mathrm{sec}$

$\begin{array}{ll} & 161.3 \\ \mathrm{DW} & 81.000 \text { usec }\end{array}$

6.00 us

$2.00000000 \mathrm{~K}$

$======$ CHANNEL $f 11=======$
NUC1

P1 9.30 use

$\begin{array}{lr}\text { PL1 } & -1.00 \mathrm{~dB} \\ \text { SF01 } & 300.1318534\end{array}$

parameters

$\begin{array}{lr}\text { SI } & 300.1300000 \mathrm{MHz}\end{array}$

WOW $\quad$ EM

$\begin{array}{lc}\text { SSB } & 0 \\ \text { LB } & 0.30 \mathrm{~Hz}\end{array}$

GB

10 NMR plot parameters

$\begin{array}{lr}\text { CX } & 21.00 \mathrm{~cm} \\ \mathrm{CY} & 8.00 \mathrm{~cm}\end{array}$

$\begin{array}{lr}\text { CY } & 8.00 \mathrm{~cm} \\ \text { F1P } & 9.000 \mathrm{ppm}\end{array}$

F1 $2701.17 \mathrm{~Hz}$

F2P $\quad-0.500 \mathrm{pp}$

$\begin{array}{ll}\text { F2 } & -150.06 \mathrm{~Hz} \\ \text { PPMCM } & 0.45238\end{array}$

$\begin{array}{lr}\text { PPMCM } & 0.45238 \mathrm{ppm} / \mathrm{cm} \\ \text { HZCM } & 135.77310 \mathrm{~Hz} / \mathrm{cm}\end{array}$ 
${ }^{13} \mathrm{C}$ NMR of 4

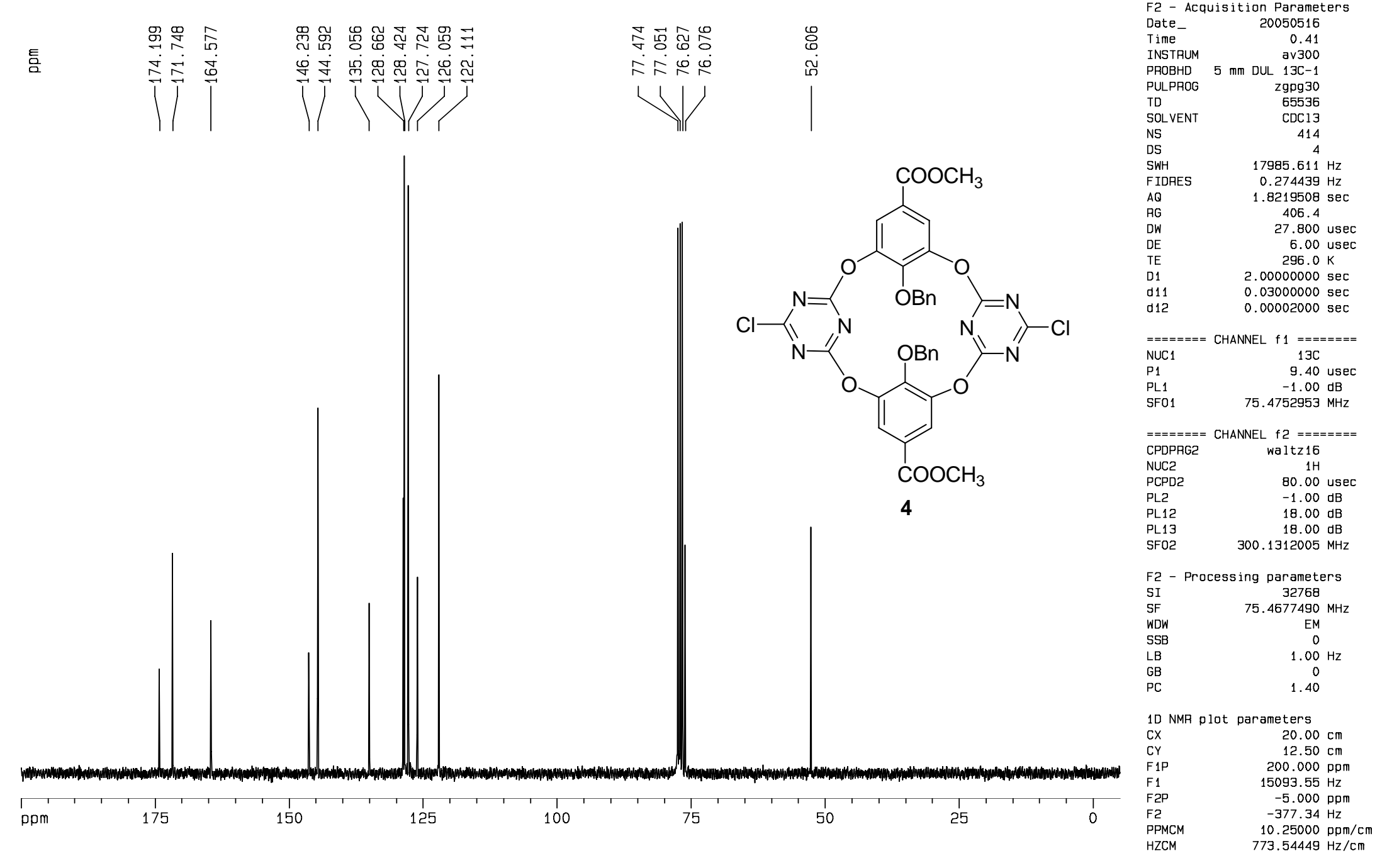


${ }^{1}$ HNMR of $\mathbf{5}$

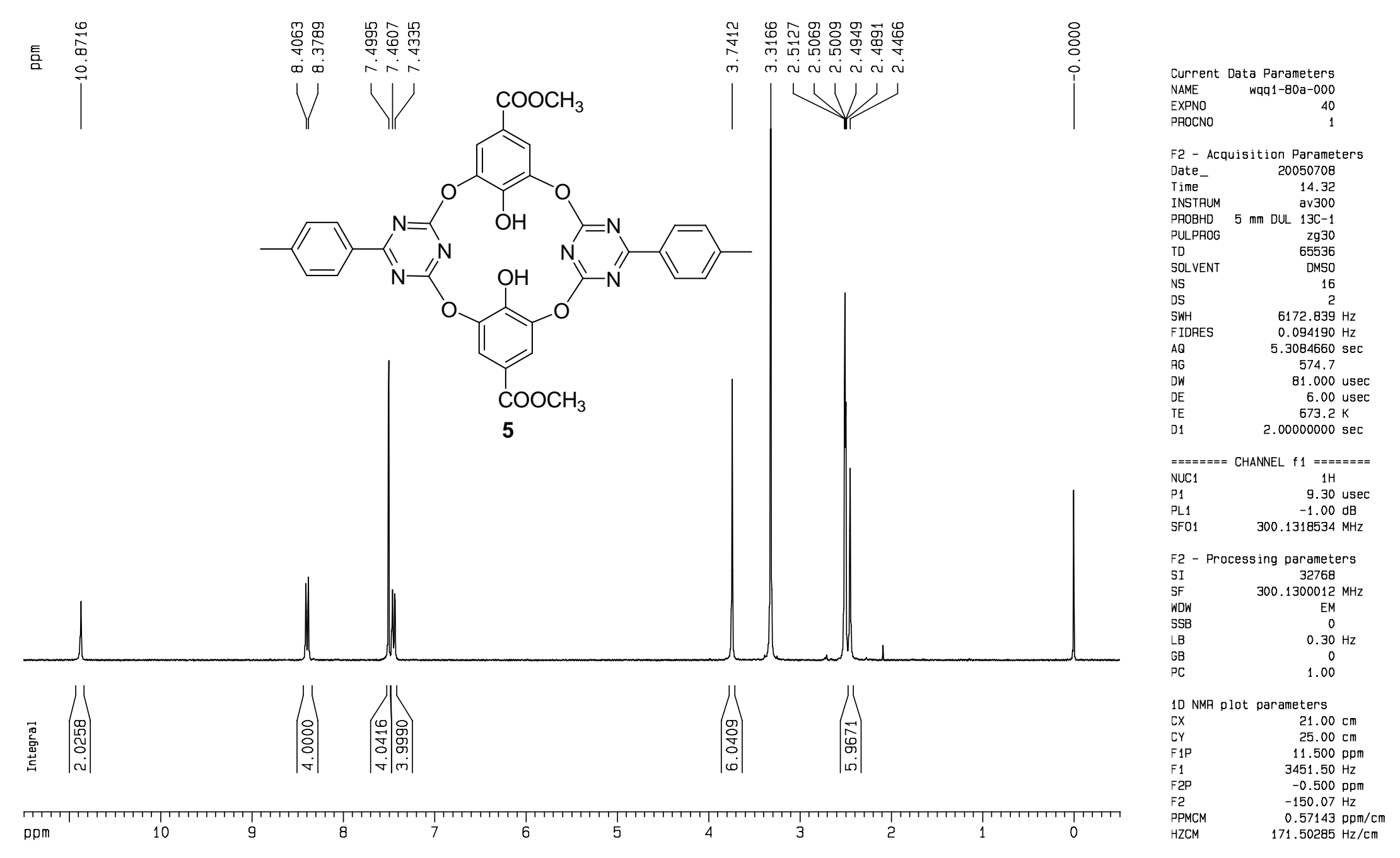


${ }^{13} \mathrm{C} \mathrm{NMR}$ of 5

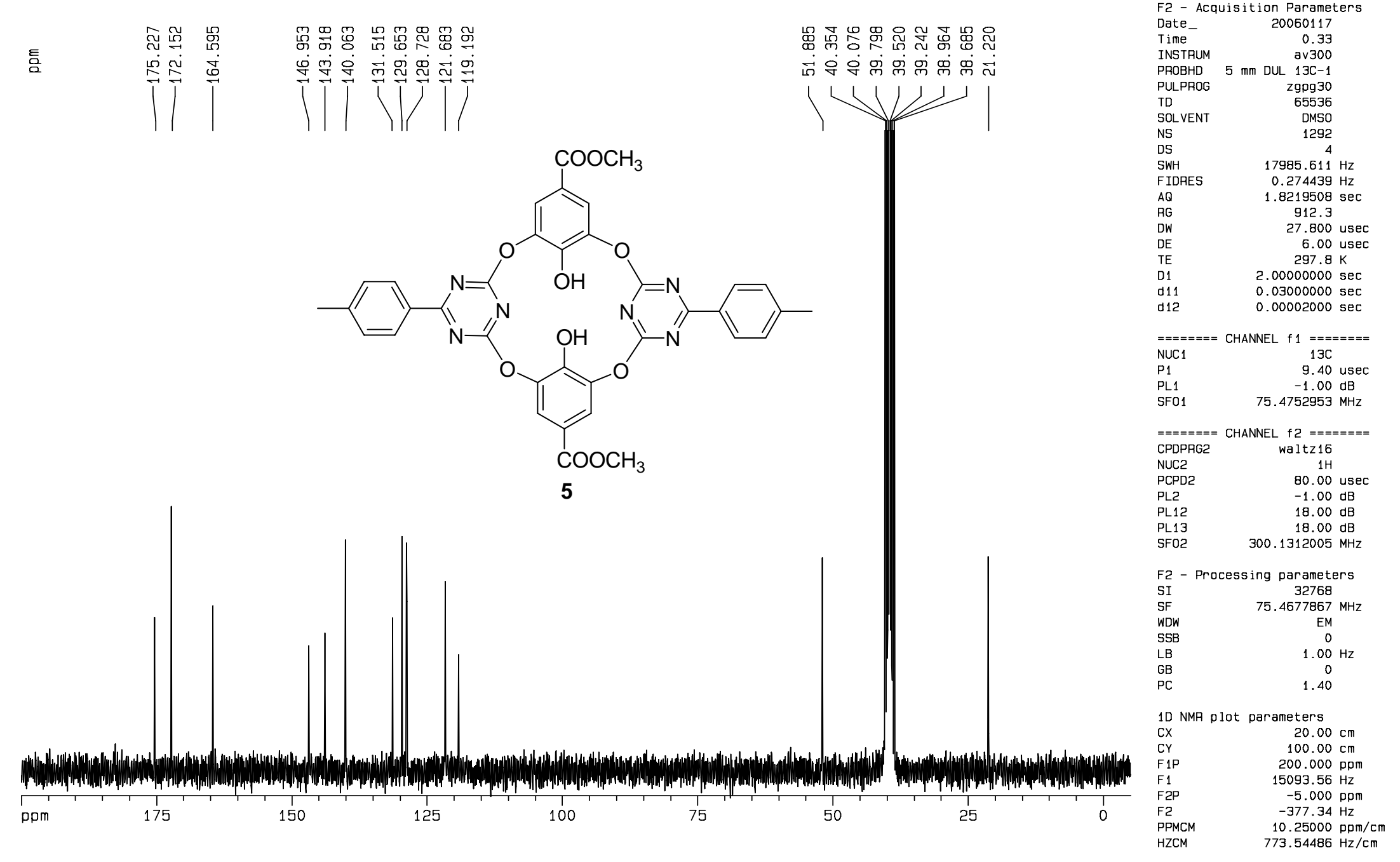




\section{${ }^{1} \mathrm{H}$ NMR of 7}
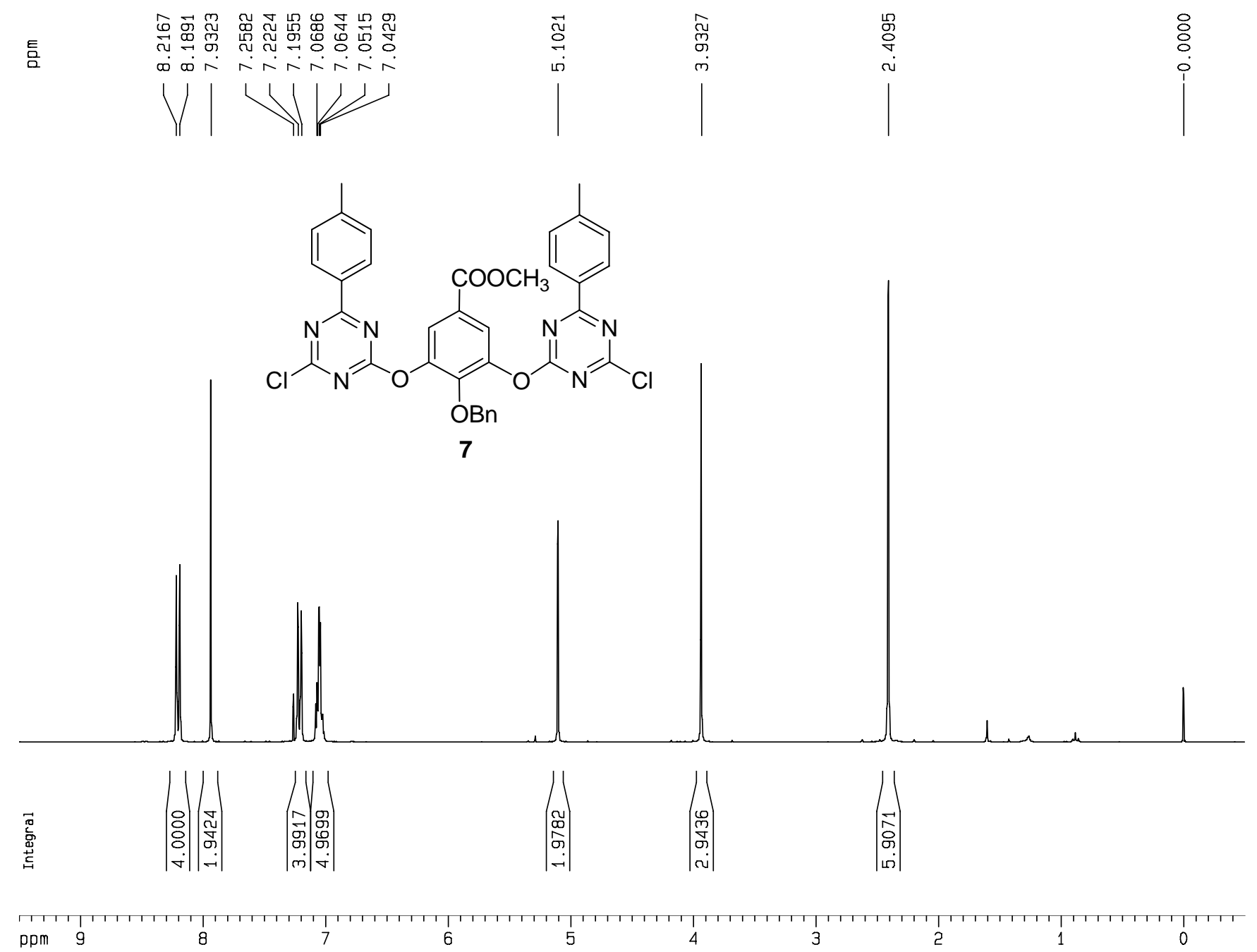

$\begin{array}{lr}\text { Current } & \text { Data Parameters } \\ \text { NAME } & \text { wqq1-86a-000 } \\ \text { EXPNO } & 20 \\ \text { PROT } & 1\end{array}$ PROCNO

F2 - Acquisition Parameters

Date $\quad 20051118$

Time $\quad 21.49$

5 PROBHD $5 \mathrm{~mm}$ DUL $13 \mathrm{C}-1$

$\begin{array}{ll}\text { TD } & 65536 \\ \text { 5OLVENT } & \text { CDC13 }\end{array}$

NS $\quad 16$

$\begin{array}{lr}\text { DS } & 2 \\ \text { SWH } & 6172.839 \mathrm{~Hz}\end{array}$

IDRES $\quad 0.094190 \mathrm{~Hz}$

AQ $\quad 5.3084660 \mathrm{sec}$

$\begin{array}{lr}\text { DW } & 81.000 \text { usec } \\ \text { DE } & 6.00 \text { usec }\end{array}$

TE $296.6 \mathrm{~K}$

D1 2.000

$=======$ CHANNEL $f 11======$
NUC1

$\begin{array}{lr}\text { P1 } & 9.30 \text { usec } \\ \text { PL1 } & -1.00 \mathrm{~dB}\end{array}$

SF01 $300.1318534 \mathrm{MHZ}$

F2 - Processing parameters

SI $\quad 32768$

WDW $\quad 300.1300059 \mathrm{MHZ}$

WDW

LB
GB

10 NMP plot paratetes

CX $\quad 21.00 \mathrm{~cm}$

F1P $\quad 9.500 \mathrm{ppm}$

F1 $2851.24 \mathrm{~Hz}$

F2P $\quad-0.500 \mathrm{ppm}$

$-150.06 \mathrm{~Hz}$

$\begin{array}{lr} & 0.47619 \mathrm{ppm} / \mathrm{cm} \\ \text { HZCM } & 142.91905 \mathrm{~Hz} / \mathrm{cm}\end{array}$ 
${ }^{13} \mathrm{C}$ NMR of 7

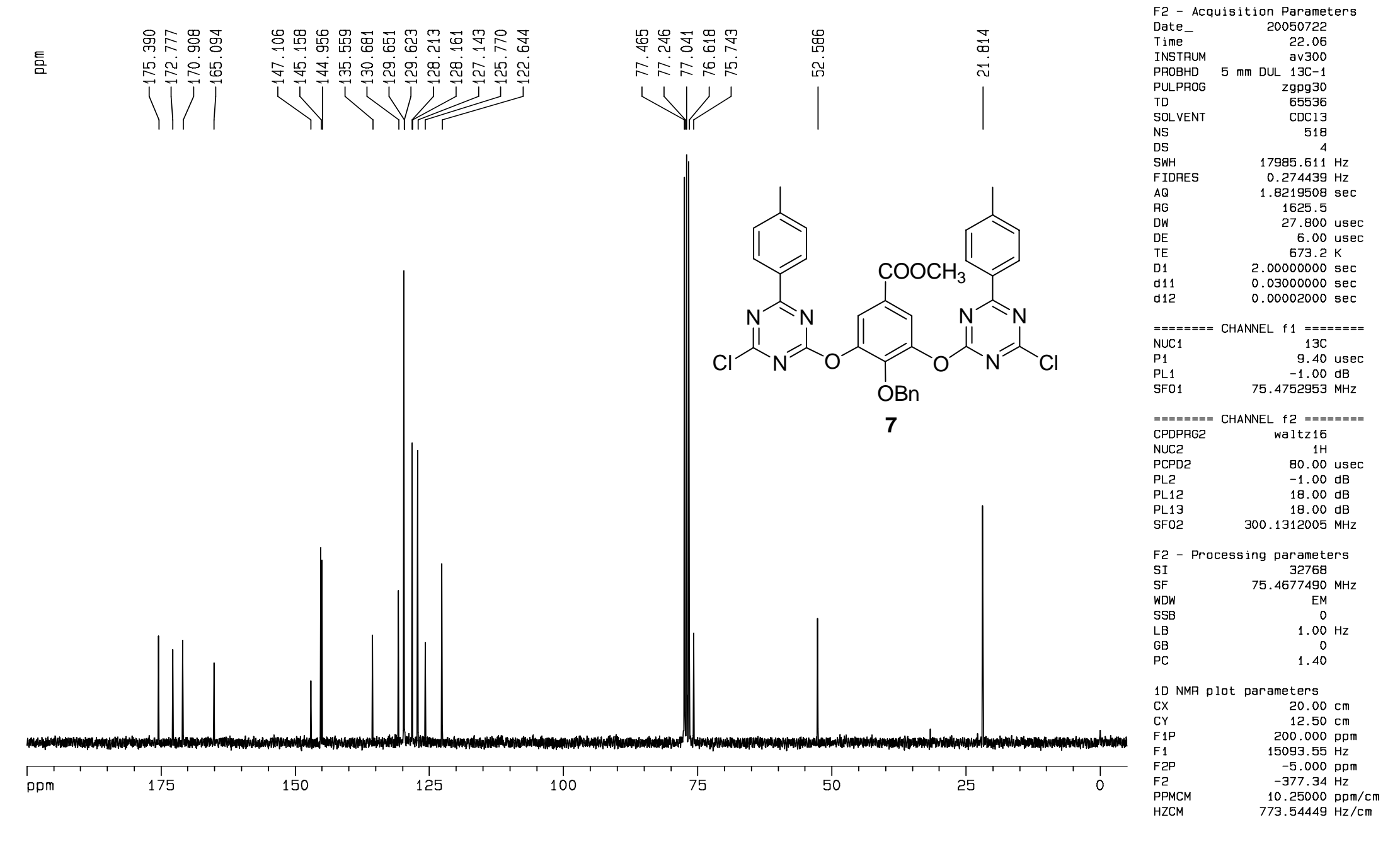




\section{${ }^{1} \mathrm{H}$ NMR of $\mathbf{8 a}$}

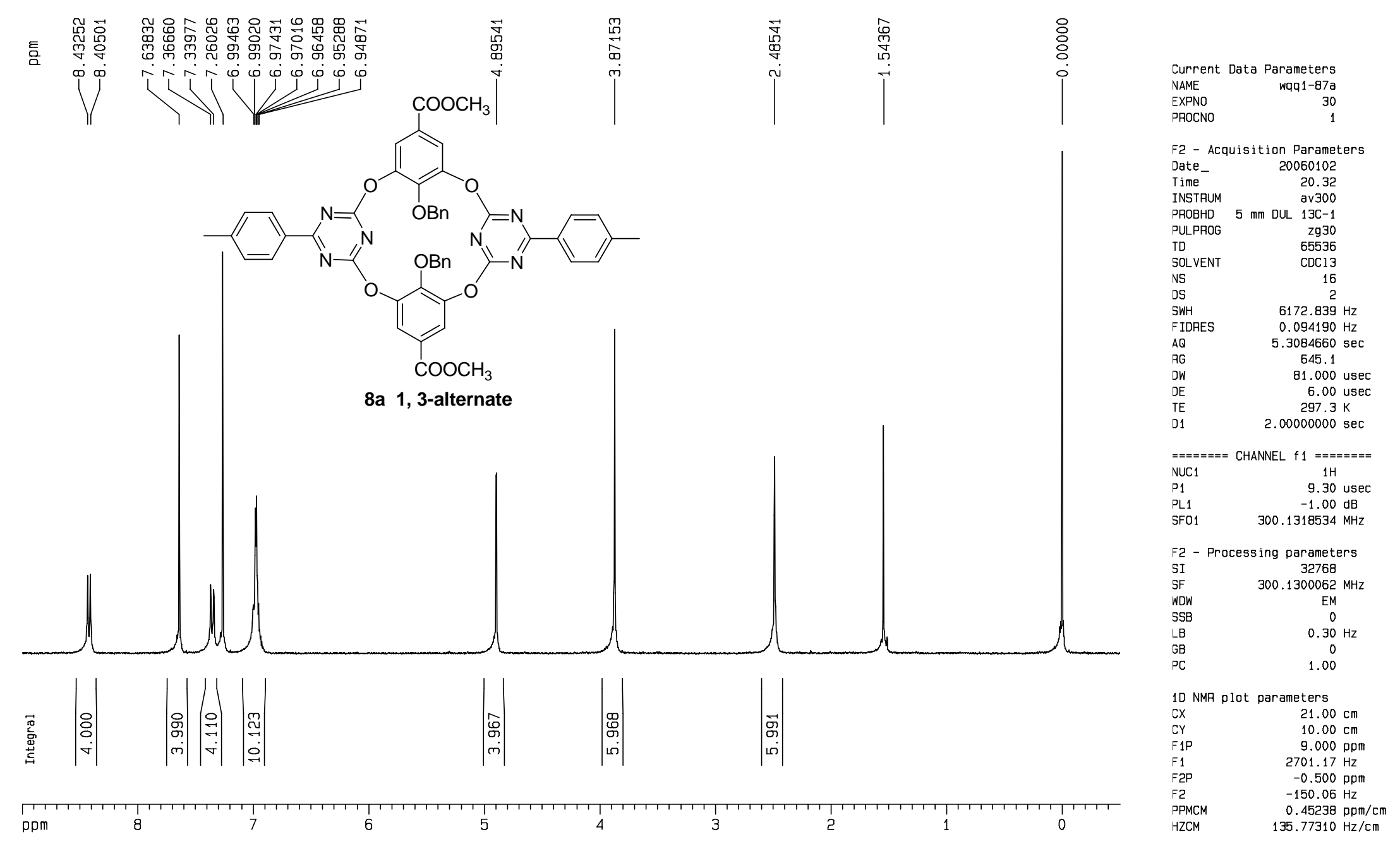


${ }^{13} \mathrm{C} \mathrm{NMR}$ of $\mathbf{8 a}$

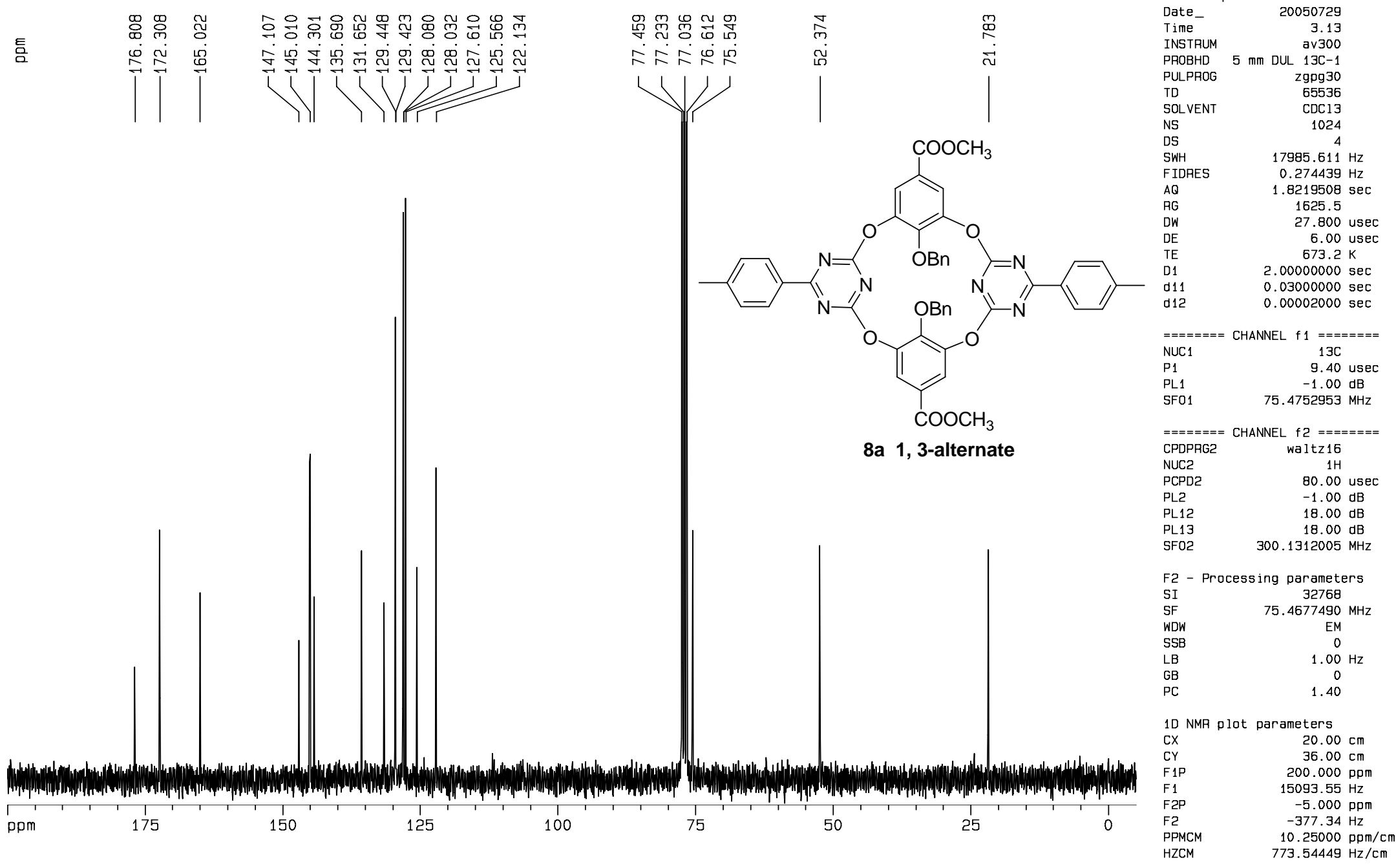


${ }^{1} \mathrm{H}$ NMR of $\mathbf{8 b}$

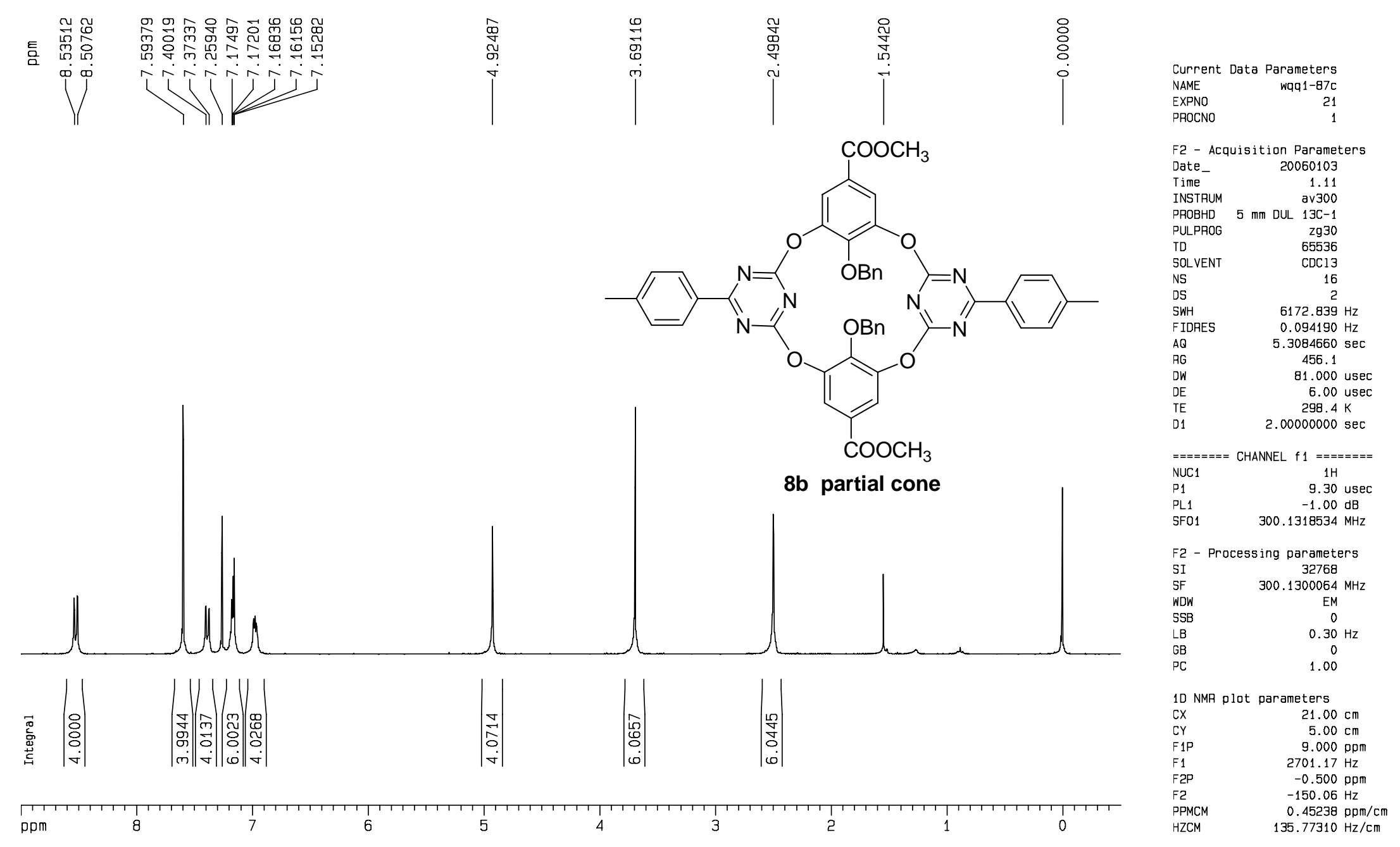


${ }^{13} \mathrm{C}$ NMR of $\mathbf{8 b}$

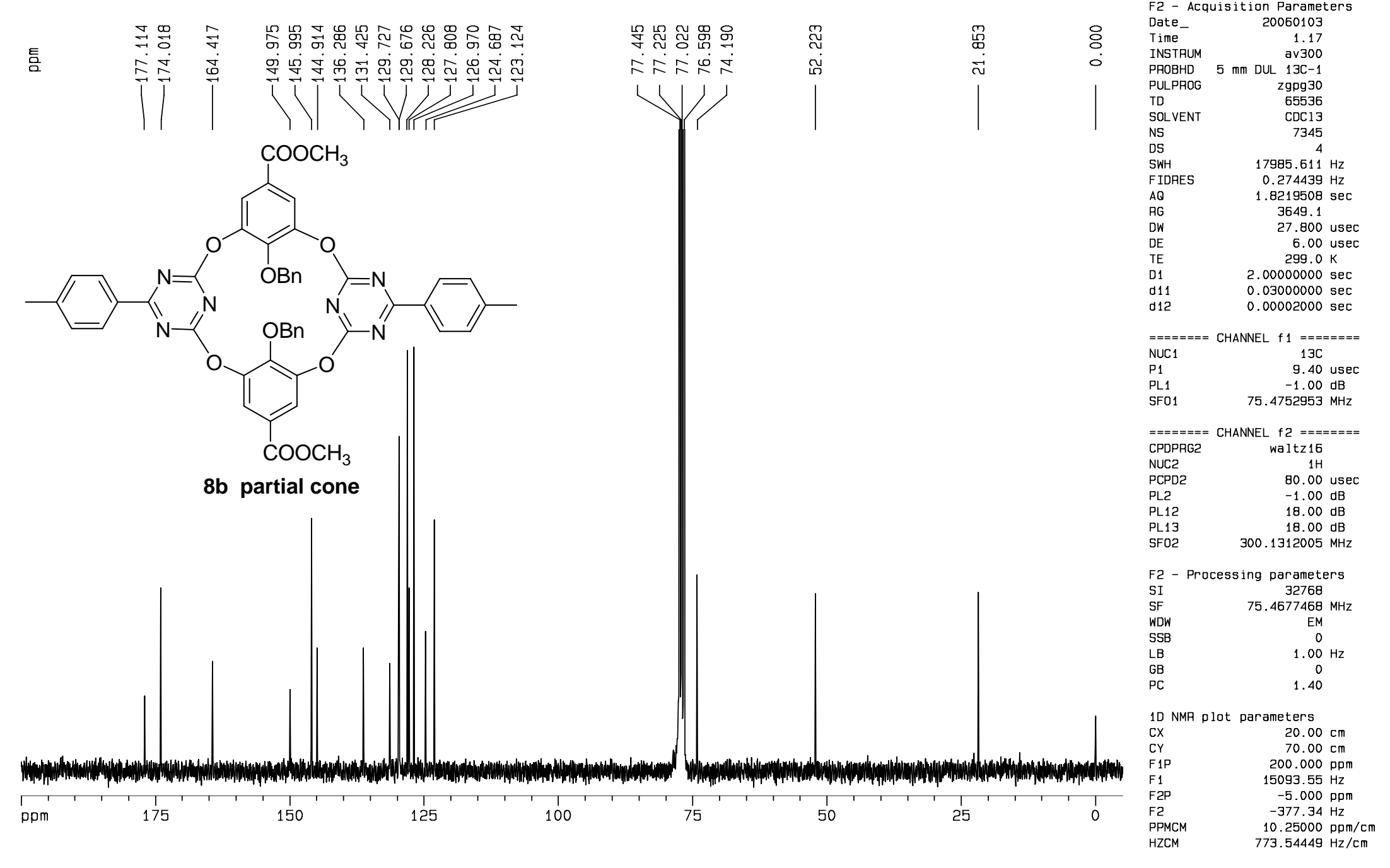


${ }^{1}$ HNMR of 9

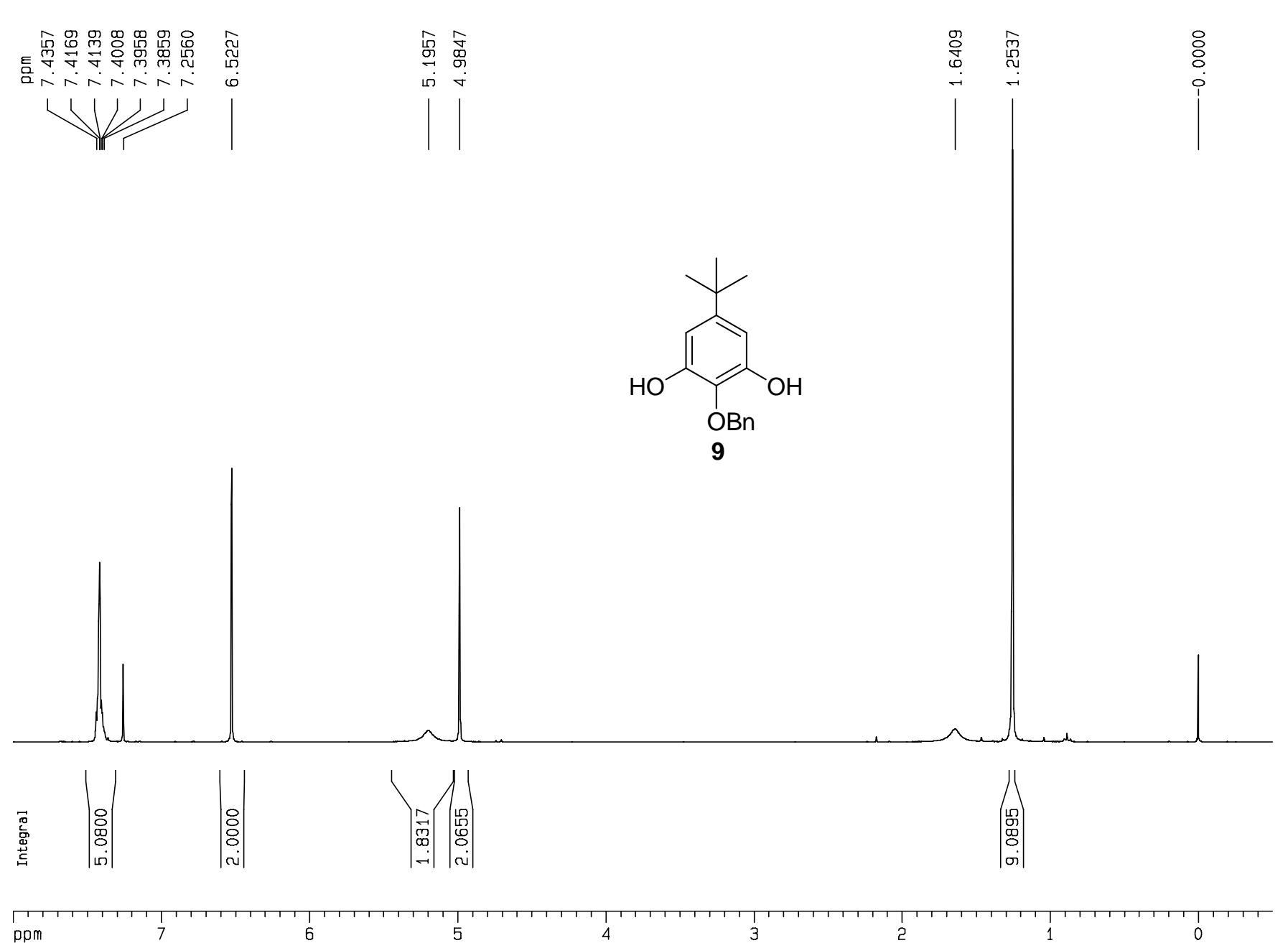
Current Data Parameters
$\begin{array}{lr}\text { NAME } & \text { WqQ3-43a } \\ \text { EXPNO } & 30\end{array}$
F2 - Acquisition Parameters
$\begin{array}{lr}\text { Date } & 20061002 \\ \text { Time } & 15.34\end{array}$
$\begin{array}{ll}15.34 \\ \text { INSTRUM } & \text { av300 }\end{array}$
PROBHD $5 \mathrm{~mm}$ DUL $13 \mathrm{C}-1$
$\begin{array}{lr}\text { PULPROG } & 2930 \\ \text { TD } & 65536\end{array}$
$\begin{array}{ll}\text { SOLVENT } & \text { CDC13 } \\ \text { NS } & 15\end{array}$
$\begin{array}{lr}\text { DS } & 2 \\ \text { SWH } & 6172.839 \mathrm{~Hz}\end{array}$
FIDRES $\quad 0.094190 \mathrm{~Hz}$
$\begin{array}{ll}A Q & 5.3084660 \mathrm{sec}\end{array}$
DW $\quad 81.000$ usec
DE $\quad 6.00$ usec
D1 $2.00000000 \mathrm{sec}$
$=======$ CHANNEL $f 1======$
NUC1
$\begin{array}{lr}\text { NuC1 } & 9.30 \text { usec } \\ \text { P1 } & -1.00 \mathrm{~dB}\end{array}$
$\begin{array}{lr}\text { PL1 } & -1.00 \mathrm{~dB} \\ \text { SF01 } & 300.1318534 \mathrm{MHz}\end{array}$
F2 - Processing parameters
$\begin{array}{ll}\text { F2 - Processing parameters } \\ \text { SI } & 32768 \\ \text { SF } & 300.1300075 \mathrm{MHz}\end{array}$
$\begin{array}{ll}\mathrm{SF} & 300.1300075 \mathrm{MHz}\end{array}$
$\begin{array}{lr}\text { WOW } & \text { EM } \\ 5 S B & 0\end{array}$
$\begin{array}{cc}\text { LB } & 0.30 \mathrm{~Hz} \\ \text { GB } & 0\end{array}$
10 NMR plot parameters
$\begin{array}{ll}\text { CX } & 22.00 \mathrm{~cm} \\ \mathrm{Cr} & 0.000 \mathrm{ppm}\end{array}$
$\begin{array}{ll}\text { CY } & 20.00 \mathrm{~cm}\end{array}$
$\begin{array}{ll}\text { F1P } & 8.000 \mathrm{ppm} \\ \text { F1 } & -0.500 \mathrm{~Hz}\end{array}$
F2P $\quad-0.500 \mathrm{~Hz}$
F2 $\quad-150.05 \mathrm{~Hz}$
$\begin{array}{lr}\text { PPMCM } & 0.38636 \mathrm{ppm} / \mathrm{cm} \\ \text { HZCM } & 115.95932 \mathrm{~Hz} / \mathrm{cm}\end{array}$

ppm

5

4

3

2

1 
${ }^{13} \mathrm{C}$ NMR of $\mathbf{9}$
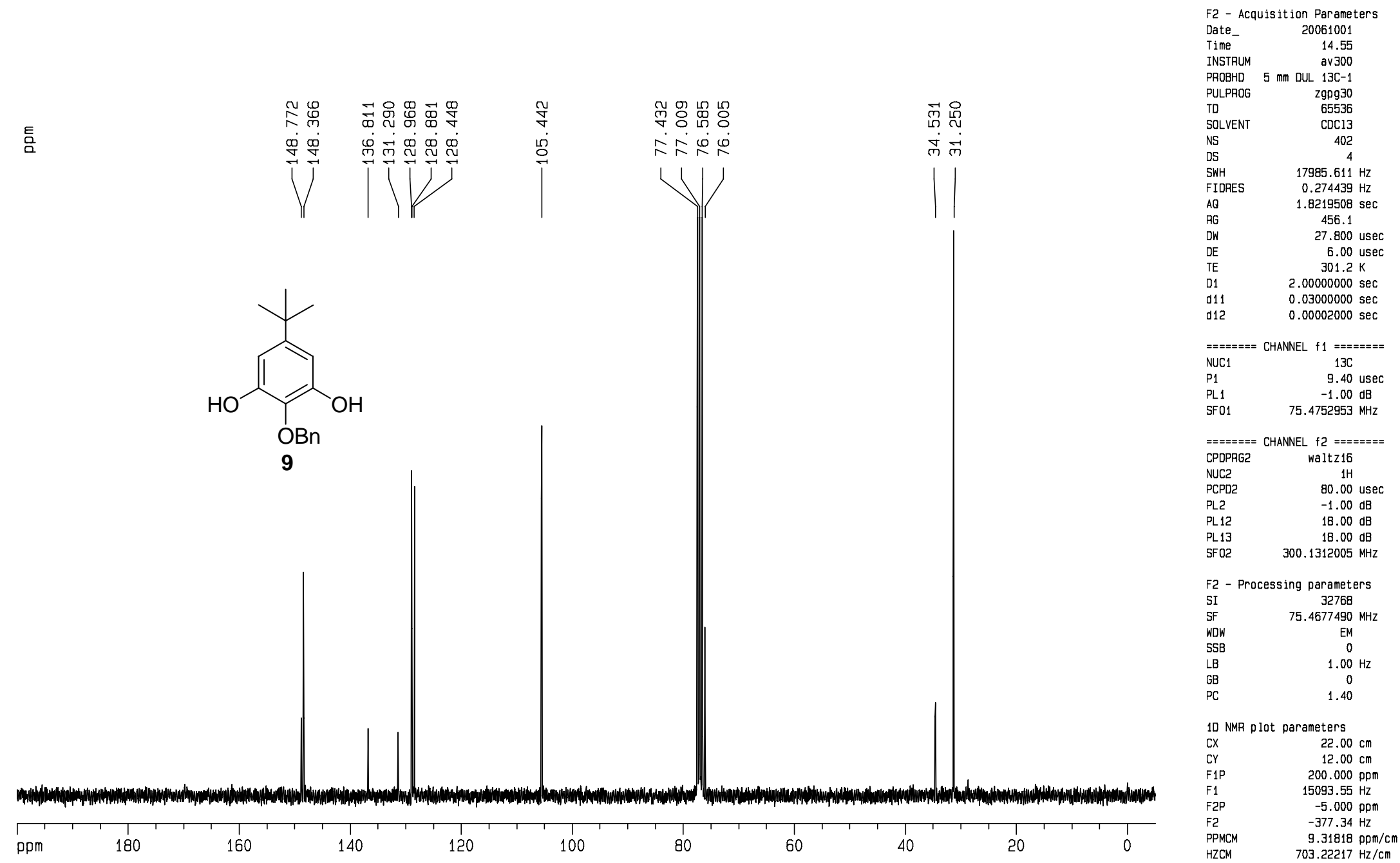
${ }^{1} \mathrm{H}$ NMR of $\mathbf{1 1}$

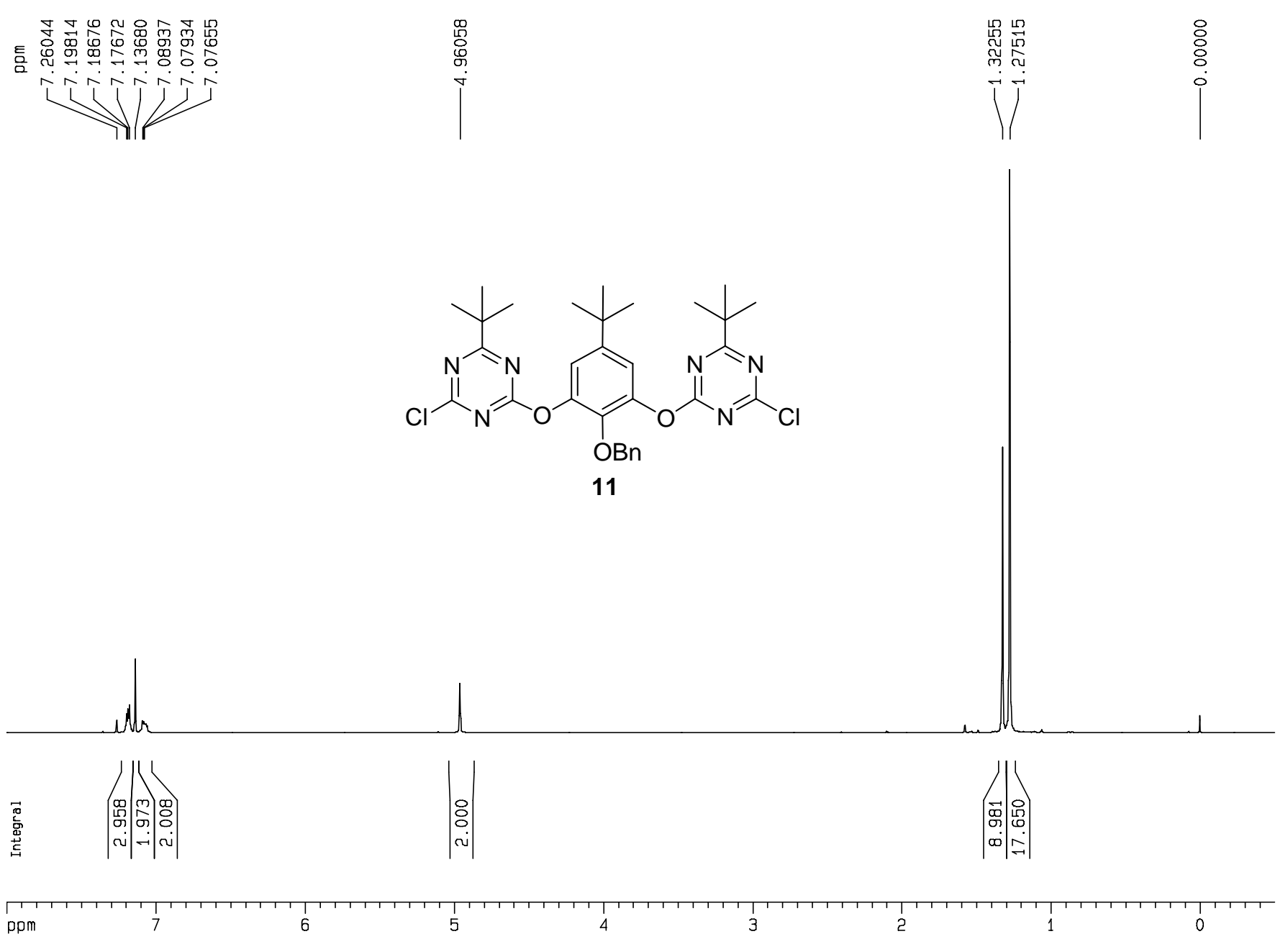

Current Data Parameters

$\begin{array}{lr}\text { NAME } & \text { WGQ3-46a } \\ \text { EXPNO } & 20\end{array}$

PROCNO

F2 - Acquisition Parameters

Date $\quad 20061010$

22.03
av300

av300
PROBHD $5 \mathrm{~mm}$ DUL $13 \mathrm{C}-1$

min DUL $13 C-1$
zg30

$\begin{array}{ll}\text { TD } & 65536 \\ \text { SOLVENT } & \text { CDC13 }\end{array}$

$\begin{array}{lr}\text { NS } & 16 \\ \text { DS } & 5\end{array}$

SWH $\quad 6172.839 \mathrm{~Hz}$

FIDRES $\quad 0.094190 \mathrm{~Hz}$

$\begin{array}{cc}\text { AQ } & 5.3084660 \mathrm{sec} \\ \text { AG } & 203.2\end{array}$

203.2
DW
81.000 usec

6.00 usec

$2.00000000 \mathrm{sec}$

$=======$ CHANNEL $f 1=======$
NUC1

P1 9.30 usec

$\begin{array}{lr}\text { PL1 } & -1.00 \mathrm{~dB} \\ \text { SF01 } & 300.1318534 \mathrm{MHz}\end{array}$

F2 - Processing parameters

$\begin{array}{lc}\text { F2 - Processing parameters } \\ \text { SI } & 32768 \\ \text { SF } & 300.1300063 \mathrm{MHz}\end{array}$

$\begin{array}{lr}\text { WOW } & \text { EM } \\ \text { SSB } & 0\end{array}$

$\begin{array}{lc}\text { SSB } & 0 \\ \text { LB } & 0.30 \mathrm{~Hz} \\ \text { GB } & 0\end{array}$

10 NMR plot parameters

$\begin{array}{ll}\text { CX } & 22.00 \mathrm{~cm} \\ \mathrm{CY} & 10.00 \mathrm{~cm}\end{array}$

$8.000 \mathrm{ppm}$
$=1$

$\begin{array}{ll}F 1 & 2401.04 \mathrm{~Hz} \\ \text { F2P } & -0.500\end{array}$

F2 $\quad-150.06 \mathrm{~Hz}$

$\begin{array}{lr}\text { PPMCM } & 0.38636 \mathrm{ppm} / \mathrm{cm} \\ \text { HZCM } & 115.95932 \mathrm{~Hz} / \mathrm{cm}\end{array}$

ppm

6

5

3

2

1 
${ }^{13} \mathrm{C}$ NMR of $\mathbf{1 1}$

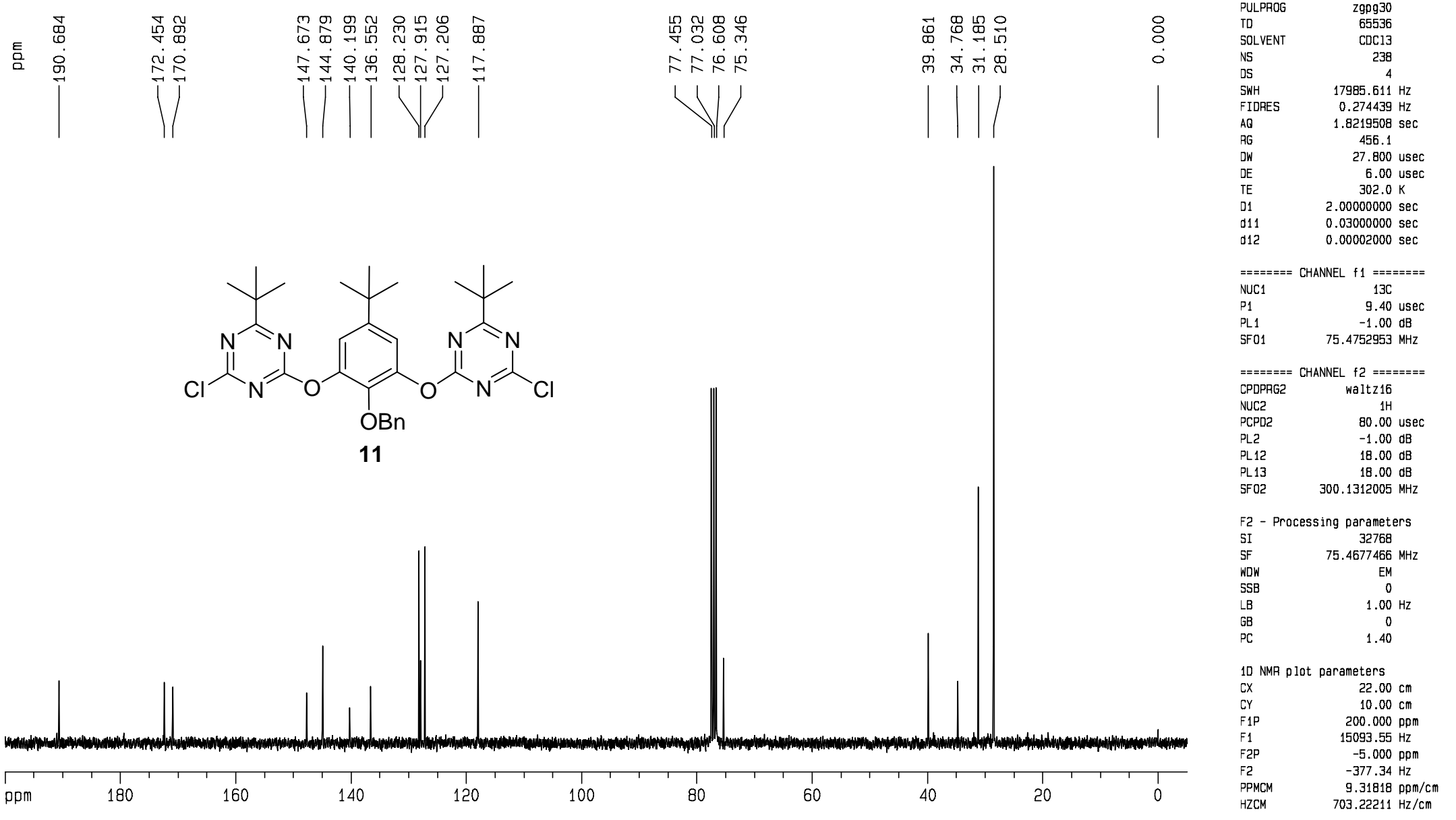


${ }^{1} \mathrm{H} N M R$ of 12a

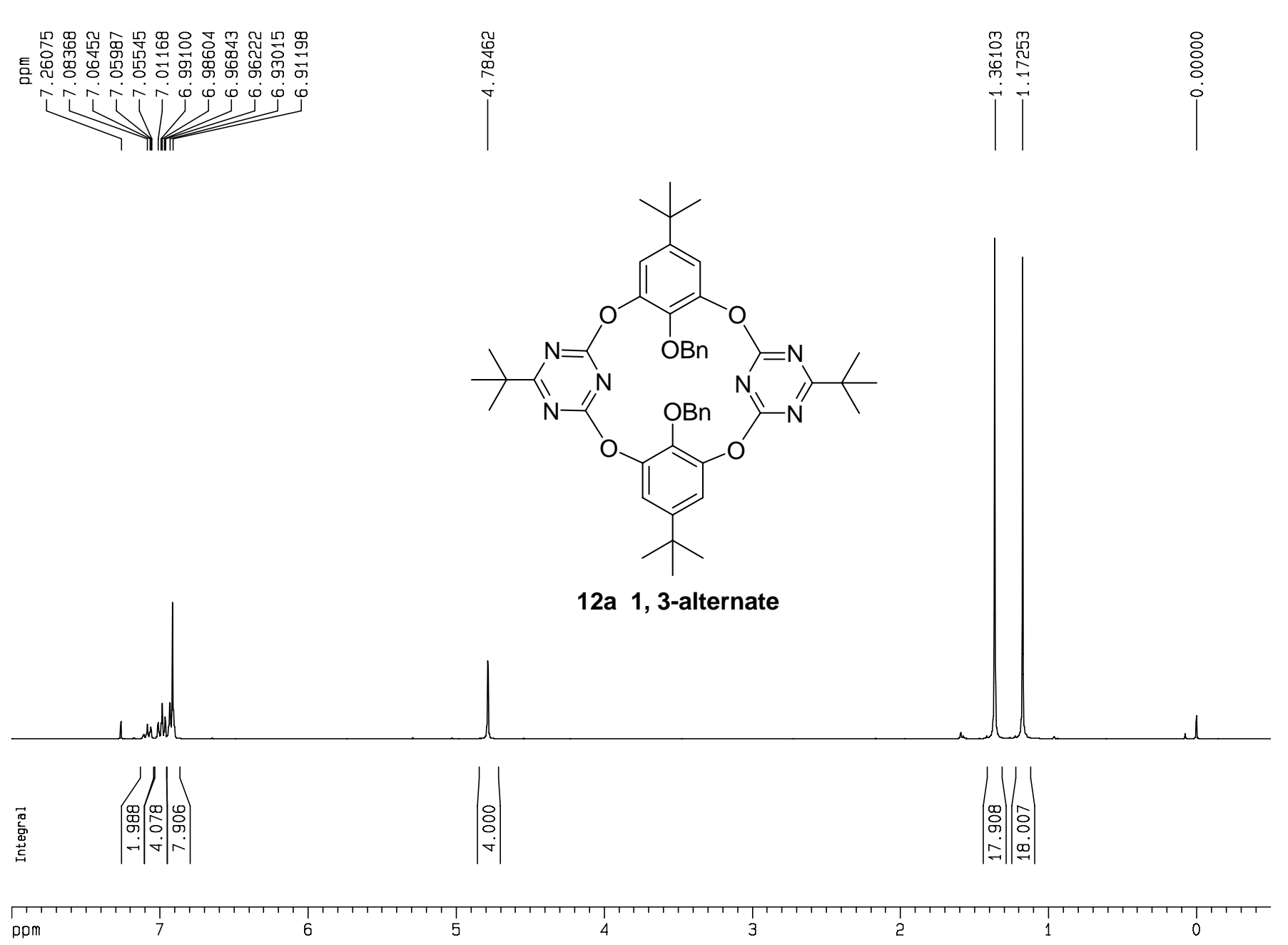


${ }^{13} \mathrm{C} \mathrm{NMR}$ of $\mathbf{1 2 a}$
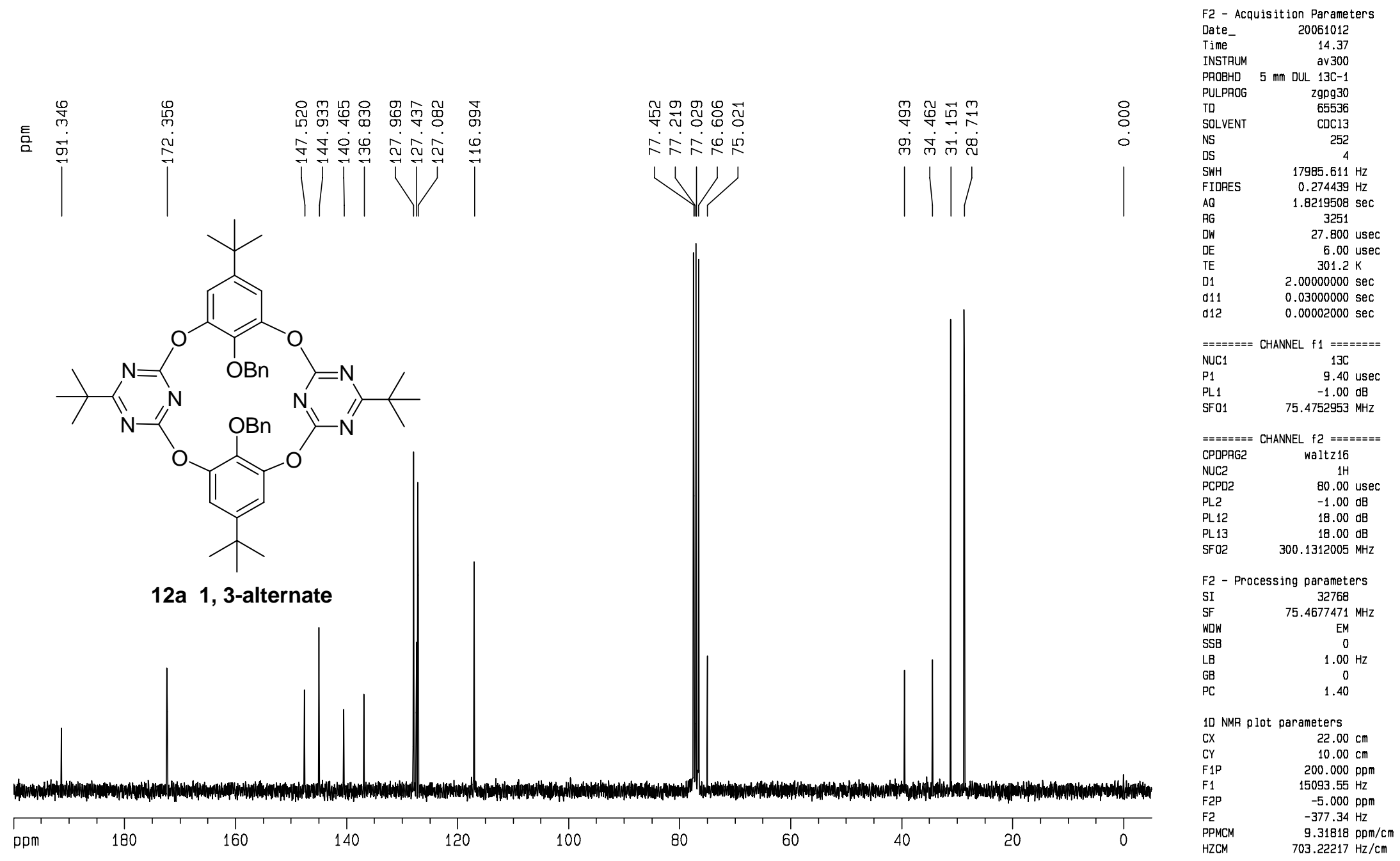
${ }^{1} \mathrm{H} N M R$ of $\mathbf{1 2 b}$

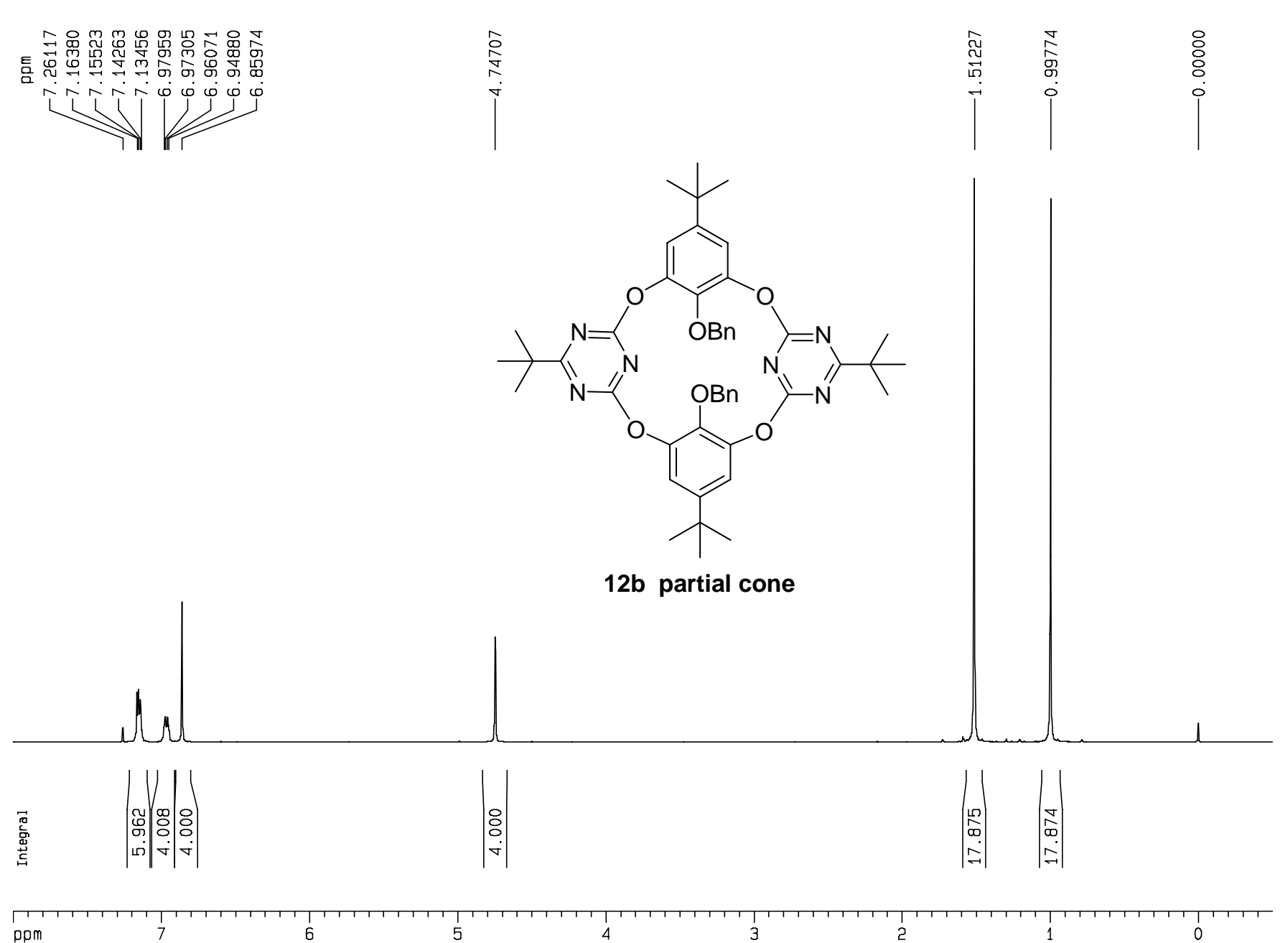
Current Data Parameters
NAME
EXPNO
F2 - Acquisition Paraneters
F2 - Acqu
Date
$\begin{array}{lr}\text { Date } & 20061016 \\ \text { Time } & 16.52\end{array}$
INSTRUM av300
PROBHD $5 \mathrm{~mm}$ DUL $13 \mathrm{C}-1$
\begin{tabular}{lr} 
PULPPOG & 29930 \\
TD & 65536 \\
\hline
\end{tabular}
$\begin{array}{lr}\text { SOLVENT } & \text { CDC13 } \\ \text { NS } & 5\end{array}$
$\begin{array}{ll}\text { DS } & 2 \\ \text { SWH } & 6172.839 \mathrm{~Hz}\end{array}$
FIDRES $\quad 0.094190 \mathrm{~Hz}$
$\begin{array}{ll}\text { AQ } & 5.3084660 \mathrm{sec} \\ \mathrm{RG} & 161.00\end{array}$
DW $\quad 81.000$ usec
DE $\quad 6.00$ usec
D1 $2.00000000 \mathrm{sec}$
$======$ CHANNEL $f 1=======$
NUC1 $1 \mathrm{H}$
$\begin{array}{lr}\text { P1 } & 9.30 \text { usec } \\ \text { Pl1 } & -1.00 \text { dB }\end{array}$
$\begin{array}{lr}\text { PL1 } & -1.00 \mathrm{~dB} \\ \text { SF01 } & 300.1318534 \mathrm{MHz}\end{array}$
F2 - Processing parameters
$\begin{array}{lc}\text { SI } & 32768 \\ \text { SF } & 300.1300059 \mathrm{MHz}\end{array}$
WDW $\quad 300.1300059 \mathrm{MHz}$
$5 S B \quad 0$
$\begin{array}{cc}L B & 0.30 \mathrm{~Hz} \\ G B & 0\end{array}$
10 NMR plot parameters
$\begin{array}{ll}\text { CX } & 22.00 \mathrm{~cm} \\ \text { CY } & \text { DP }\end{array}$
$\begin{array}{ll}\text { CY } & 10.00 \mathrm{~cm}\end{array}$
$\begin{array}{ll}\text { F1P } & 8.000 \mathrm{ppm} \\ \text { F1 } & -0.500 \mathrm{pm}\end{array}$
$\begin{array}{ll}F 1 & 2401.04 \mathrm{~Hz} \\ \text { F2P } & -0.500 \mathrm{ppm} \\ \text { F2 } & -150.05 \mathrm{~Hz}\end{array}$
$\begin{array}{lr}\text { PPMCM } & 0.38636 \mathrm{ppm} / \mathrm{cm} \\ \text { HZCM } & 115.95932 \mathrm{~Hz} / \mathrm{cm}\end{array}$

ppm 
${ }^{13} \mathrm{C} \mathrm{NMR}$ of $\mathbf{1 2 b}$
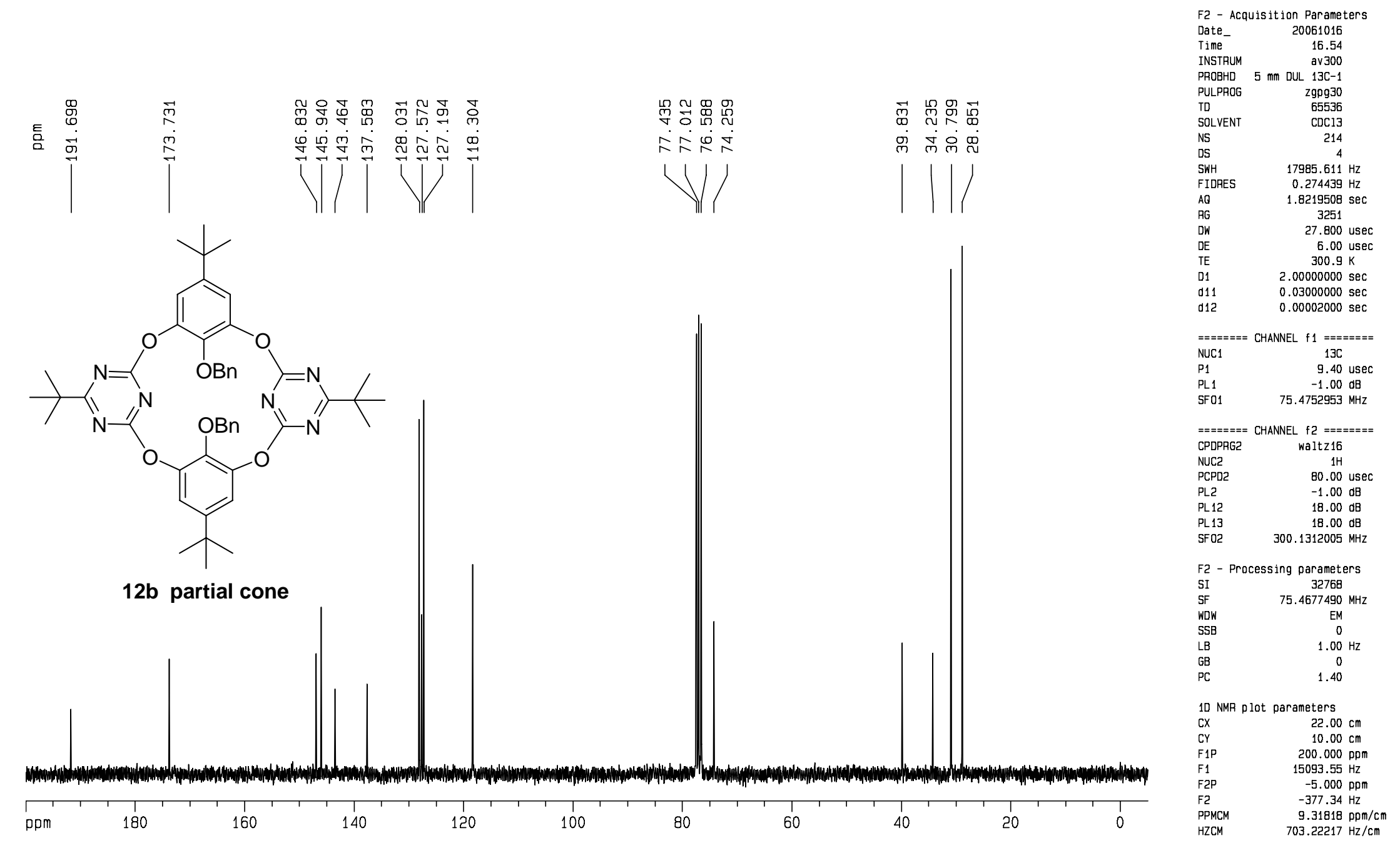
${ }^{1} \mathrm{H}$ NMR of $\mathbf{1 3}$
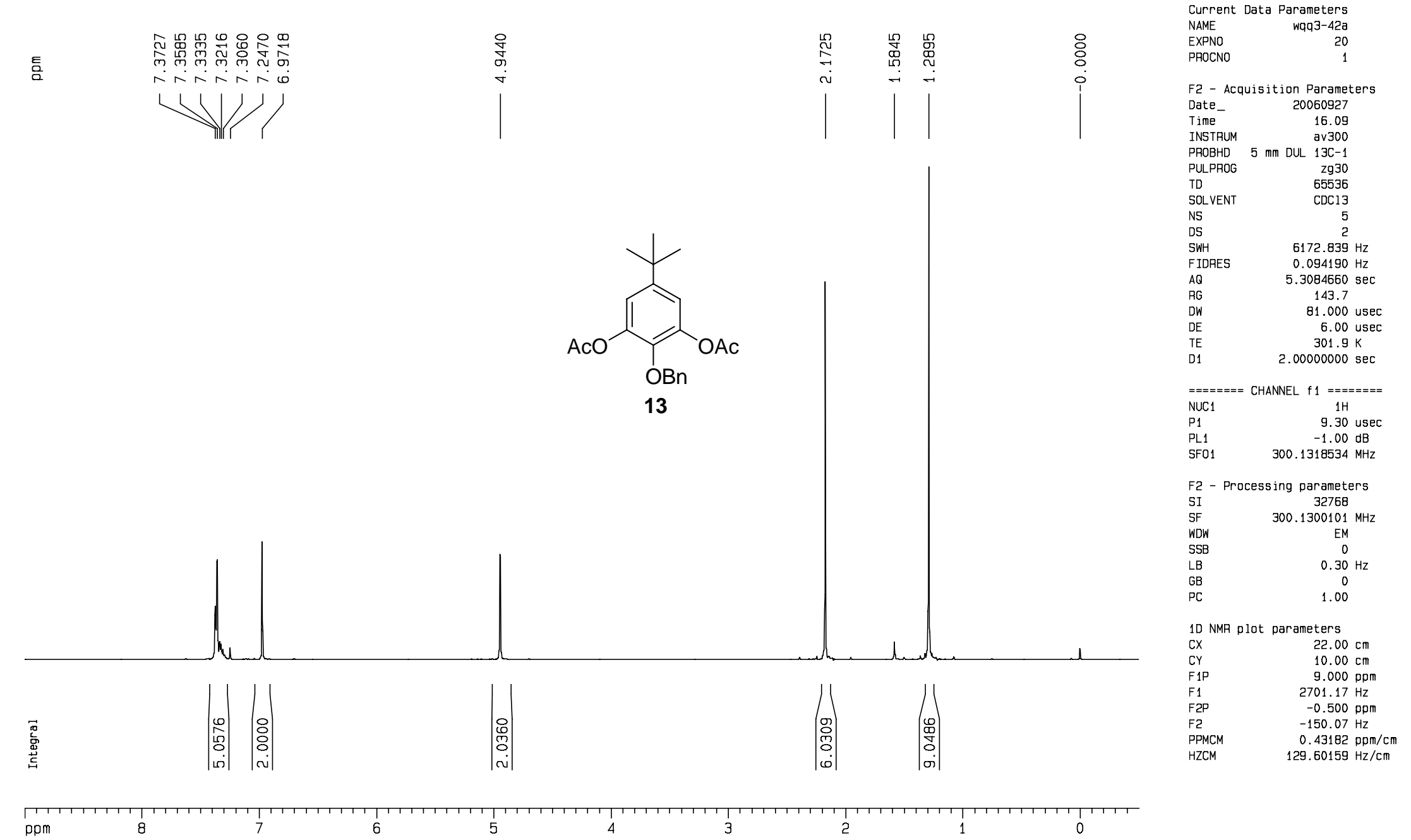
${ }^{13} \mathrm{C}$ NMR of $\mathbf{1 3}$
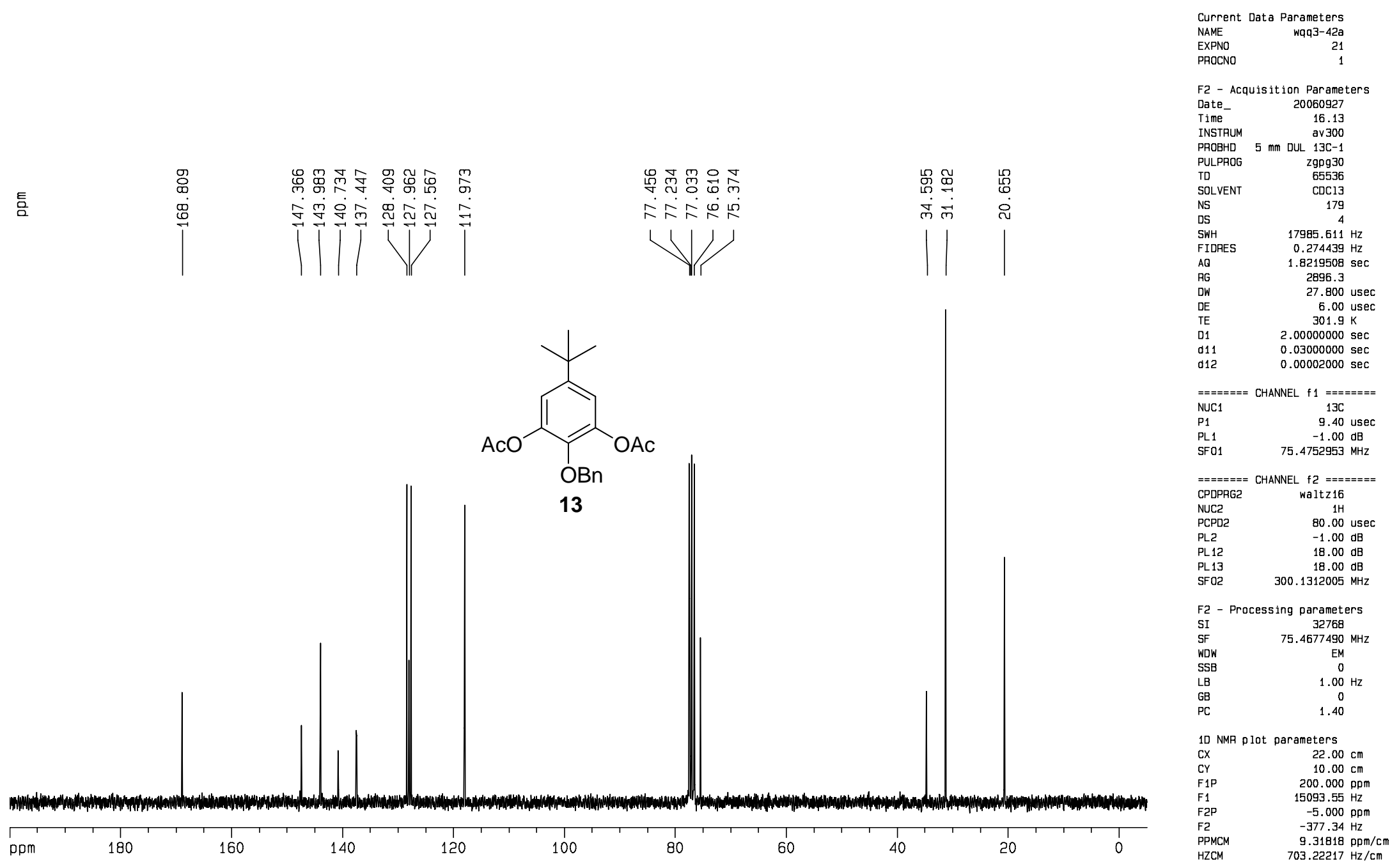
X-Ray structure of $4,8 a, 8 b, 12 a$ and $12 b$
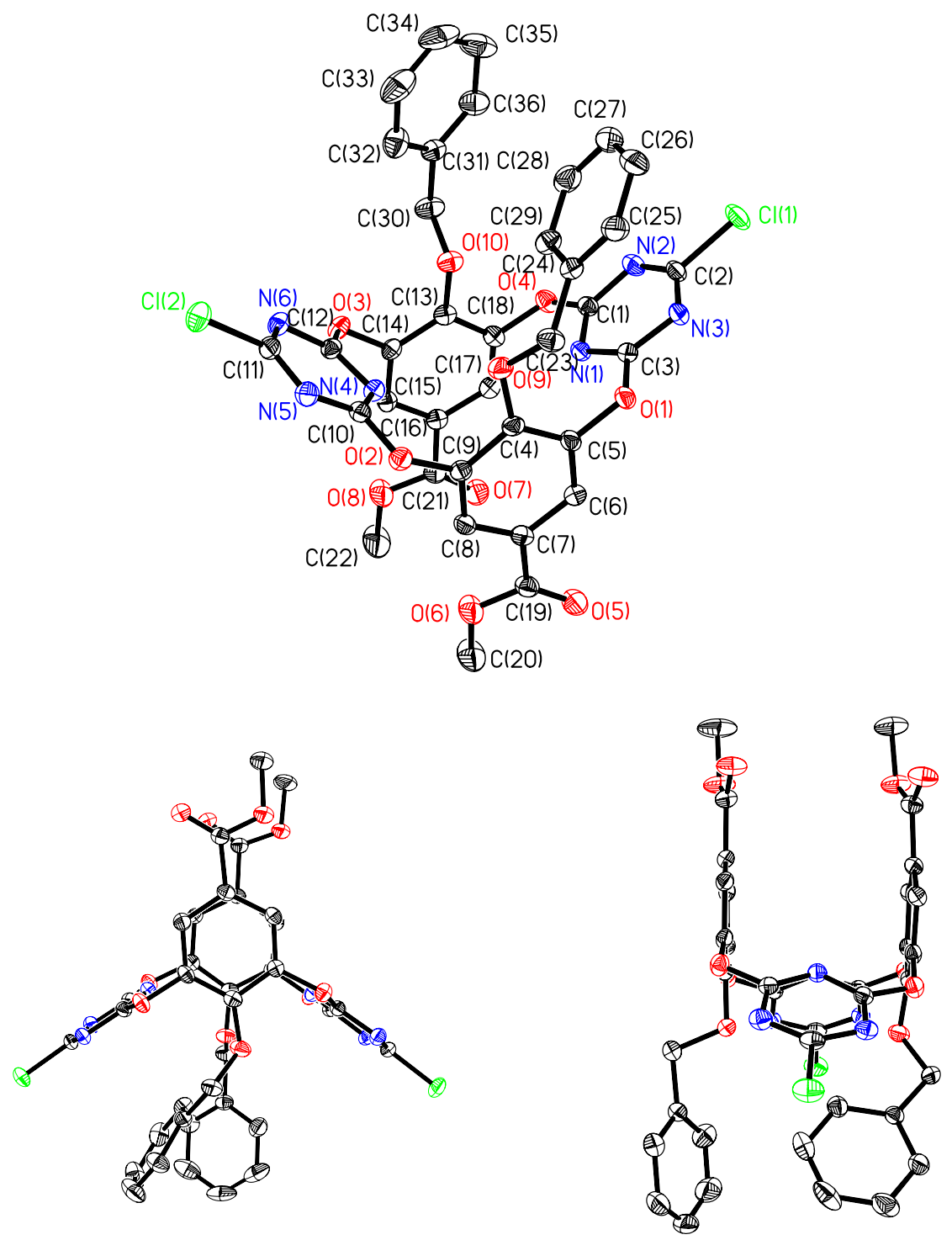

Figure S1. X-ray structure of 4: top and side views. Distances between $\mathrm{O}(1)$ and $\mathrm{C}(3), \mathrm{O}(1)$ and $\mathrm{C}(5), \mathrm{O}(2)$ and $\mathrm{C}(10), \mathrm{O}(2)$ and $\mathrm{C}(9), \mathrm{O}(3)$ and $\mathrm{C}(12)$, $\mathrm{O}(3)$ and $\mathrm{C}(14), \mathrm{O}(4)$ and $\mathrm{C}(1), \mathrm{O}(4)$ and $\mathrm{C}(18)$ are 1.339, 1.410, 1.342, $1.412,1.343,1.410,1.340,1.406 \AA$, respectively 

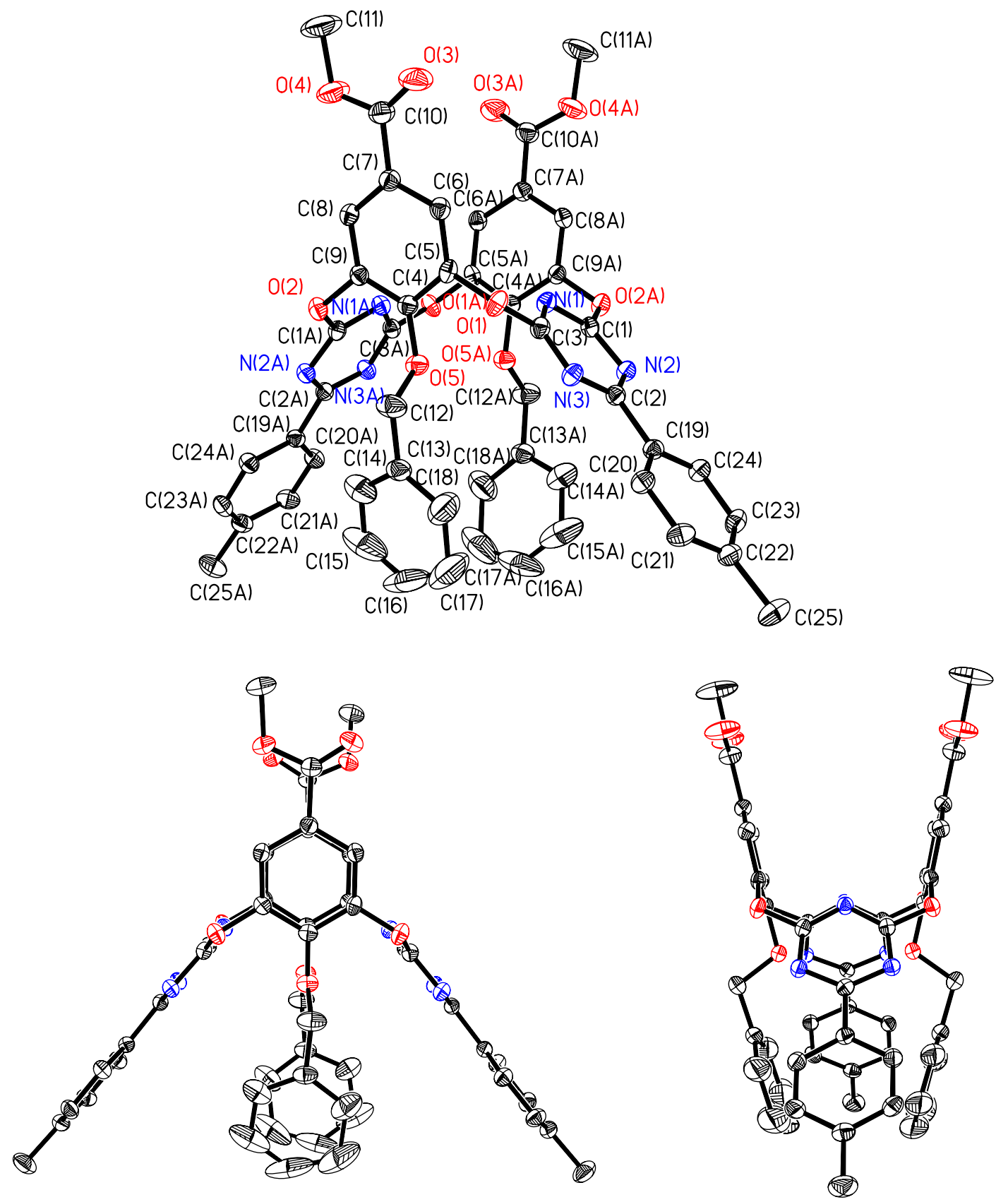

Figure S2. X-ray structure of 8a: top and side views. Distances between $\mathrm{O}(1)$ and $\mathrm{C}(3), \mathrm{O}(1)$ and $\mathrm{C}(5), \mathrm{O}(2)$ and $\mathrm{C}(1 \mathrm{~A}), \mathrm{O}(2)$ and $\mathrm{C}(9), \mathrm{O}(1 \mathrm{~A})$ and $\mathrm{C}(3 \mathrm{~A})$, $\mathrm{O}(1 \mathrm{~A})$ and $\mathrm{C}(5 \mathrm{~A}), \mathrm{O}(2 \mathrm{~A})$ and $\mathrm{C}(1), \mathrm{O}(2 \mathrm{~A})$ and $\mathrm{C}(9 \mathrm{~A})$ are 1.352, 1.403, $1.353,1.401,1.352,1.403,1.353,1.401 \AA$, respectively 


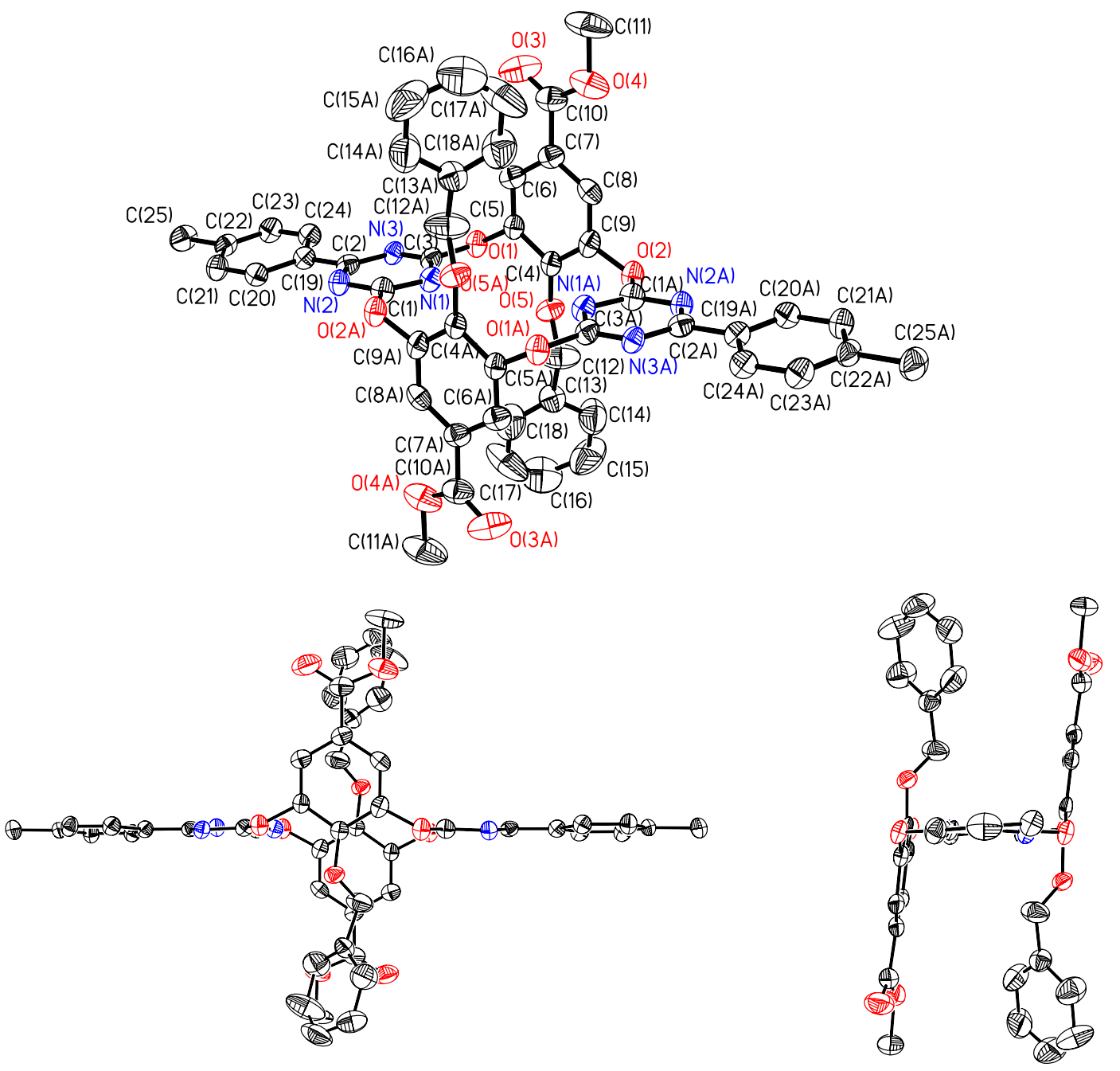

Figure S3. X-ray structure of $\mathbf{8 b}$ : top and side views. Distances between $\mathrm{O}(1)$ and $\mathrm{C}(3), \mathrm{O}(1)$ and $\mathrm{C}(5), \mathrm{O}(2)$ and $\mathrm{C}(1 \mathrm{~A}), \mathrm{O}(2)$ and $\mathrm{C}(9), \mathrm{O}(1 \mathrm{~A})$ and $\mathrm{C}(3 \mathrm{~A})$, $\mathrm{O}(1 \mathrm{~A})$ and $\mathrm{C}(5 \mathrm{~A}), \mathrm{O}(2 \mathrm{~A})$ and $\mathrm{C}(1), \mathrm{O}(2 \mathrm{~A})$ and $\mathrm{C}(9 \mathrm{~A})$ are 1.352, 1.406, $1.380,1.397,1.352,1.406,1.380,1.397 \AA$, respectively 

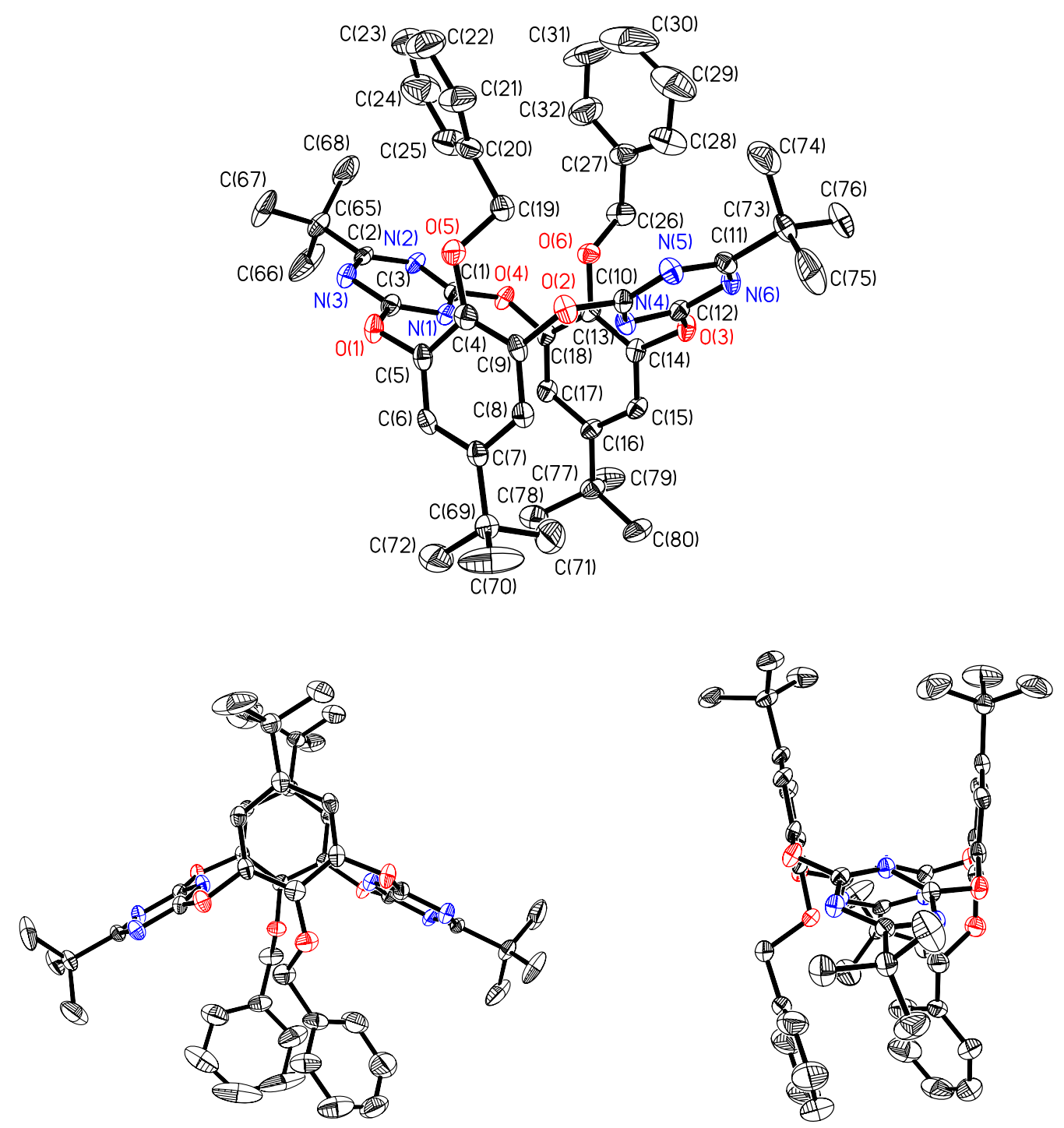

Figure S4. X-ray structure of 12a: top and side views. Distances between $\mathrm{O}(1)$ and $\mathrm{C}(3), \mathrm{O}(1)$ and $\mathrm{C}(5), \mathrm{O}(2)$ and $\mathrm{C}(10), \mathrm{O}(2)$ and $\mathrm{C}(9), \mathrm{O}(3)$ and $\mathrm{C}(12), \mathrm{O}(3)$ and $\mathrm{C}(14), \mathrm{O}(4)$ and $\mathrm{C}(1), \mathrm{O}(4)$ and $\mathrm{C}(18)$ are 1.340, 1.411, $1.355,1.410,1.348,1.410,1.349,1.410 \AA$, respectively 

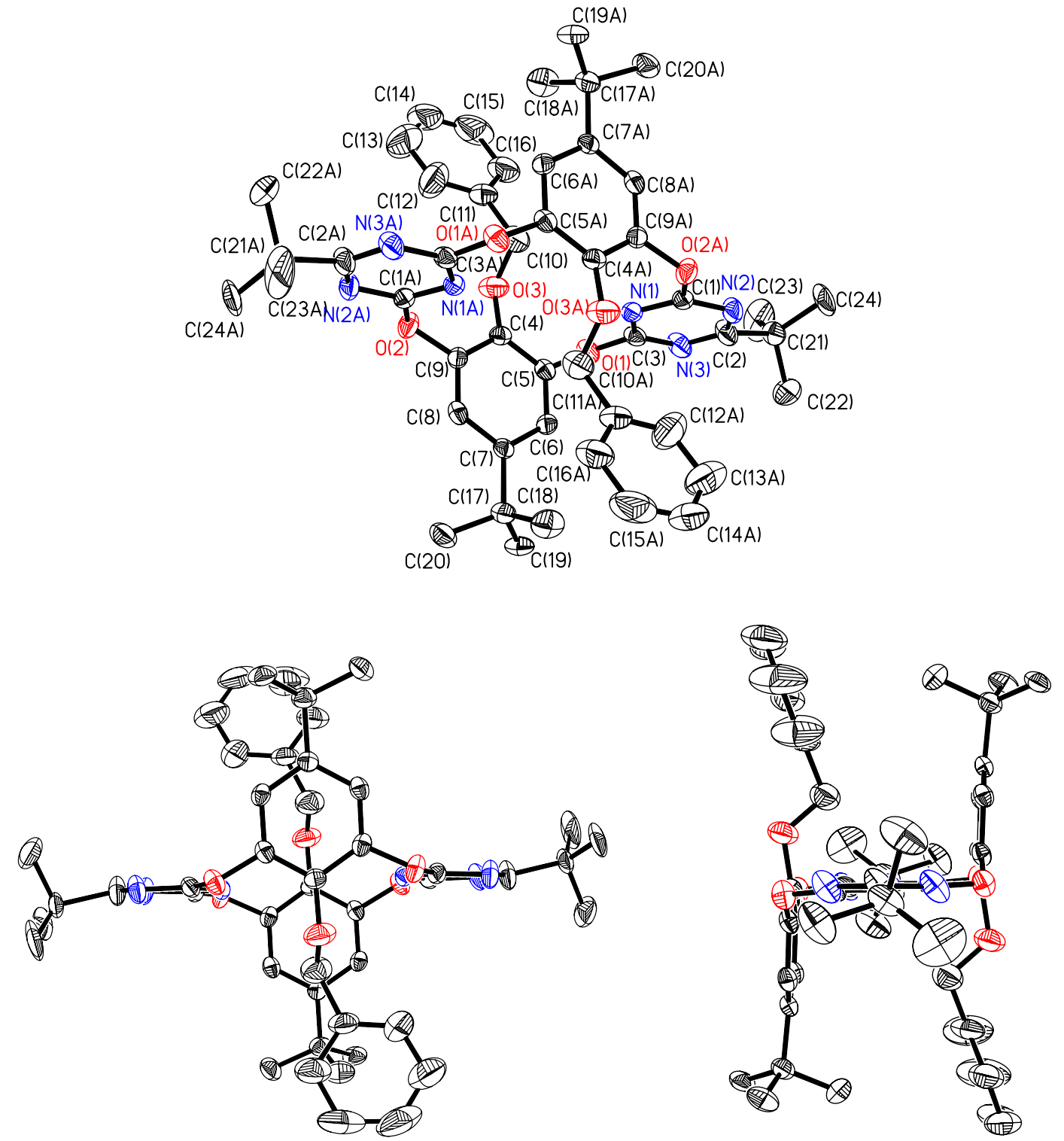

Figure S5. X-ray structure of $\mathbf{1 2 b}$ : top and side views. Distances between $\mathrm{O}(1)$ and $\mathrm{C}(3), \mathrm{O}(1)$ and $\mathrm{C}(5), \mathrm{O}(2)$ and $\mathrm{C}(1 \mathrm{~A}), \mathrm{O}(2)$ and $\mathrm{C}(9), \mathrm{O}(1 \mathrm{~A})$ and $\mathrm{C}(3 \mathrm{~A}), \mathrm{O}(1 \mathrm{~A})$ and $\mathrm{C}(5 \mathrm{~A}), \mathrm{O}(2 \mathrm{~A})$ and $\mathrm{C}(1), \mathrm{O}(2 \mathrm{~A})$ and $\mathrm{C}(9 \mathrm{~A})$ are 1.356, $1.410,1.342,1.407,1.356,1.410,1.342,1.407 \AA$, respectively 
Table S1 Crystal data and structure refinement for 4, 8a, 8b, 12a and 12b

\begin{tabular}{|c|c|c|c|c|c|}
\hline Compound & 4 & $8 a$ & $\mathbf{8 b} \cdot \mathrm{CH}_{3} \mathrm{COCH}_{3}$ & $12 a$ & 12b $\cdot \mathrm{CH}_{2} \mathrm{Cl}_{2}$ \\
\hline Empirical formula & $\mathrm{C}_{36} \mathrm{H}_{24} \mathrm{Cl}_{2} \mathrm{~N}_{6} \mathrm{O}_{10}$ & $\mathrm{C}_{50} \mathrm{H}_{38} \mathrm{~N}_{6} \mathrm{O}_{10}$ & $\mathrm{C}_{53} \mathrm{H}_{44} \mathrm{~N}_{6} \mathrm{O}_{11}$ & $\mathrm{C}_{48} \mathrm{H}_{54} \mathrm{~N}_{6} \mathrm{O}_{6}$ & $\mathrm{C}_{49} \mathrm{H}_{54} \mathrm{Cl}_{2} \mathrm{~N}_{6} \mathrm{O}_{6}$ \\
\hline$M_{r}$ & 771.51 & 882.86 & 940.94 & 810.97 & 893.88 \\
\hline crystal size, $\mathrm{mm}^{3}$ & $0.80 \times 0.33 \times 0.27$ & $0.77 \times 0.39 \times 0.02$ & $0.77 \times 0.35 \times 0.05$ & $0.81 \times 0.56 \times 0.43$ & $0.90 \times 0.63 \times 0.45$ \\
\hline crystal system & Triclinic & Monoclinic & Triclinic & Triclinic & Monoclinic \\
\hline space group & P-1 & $\mathrm{C} 2 / \mathrm{c}$ & P-1 & $\mathrm{P}-1$ & $\mathrm{C} 2 / \mathrm{c}$ \\
\hline $\mathrm{a}[\AA]$ & $9.3832(19)$ & $9.3404(19)$ & $13.358(3)$ & $14.451(3)$ & $14.890(3)$ \\
\hline $\mathrm{b}[\AA]$ & $13.585(3)$ & $27.574(6)$ & $13.955(3)$ & $17.782(4)$ & $17.963(4)$ \\
\hline $\mathrm{c}[\AA]$ & $15.427(3)$ & $17.783(4)$ & $15.064(3)$ & $19.083(4)$ & $18.365(4)$ \\
\hline$\alpha[\operatorname{deg}]$ & $109.12(3)$ & 90.00 & $62.93(3)$ & $89.89(3)$ & 90.00 \\
\hline$\beta[\operatorname{deg}]$ & $94.12(3)$ & $102.76(3)$ & $81.83(3)$ & $107.15(3)$ & $91.79(3)$ \\
\hline$\gamma[\mathrm{deg}]$ & $106.63(3)$ & 90.00 & $69.41(3)$ & $90.21(3)$ & 90.00 \\
\hline $\mathrm{V}\left[\AA^{3}\right]$ & $1750.2(6)$ & $4466.9(16)$ & $2340.3(8)$ & $4685.7(16)$ & $4909.7(17)$ \\
\hline $\mathrm{d}\left[\mathrm{g} / \mathrm{cm}^{3}\right]$ & 1.464 & 1.313 & 1.335 & 1.150 & 1.209 \\
\hline Z & 2 & 4 & 2 & 4 & 4 \\
\hline $\mathrm{T}[\mathrm{K}]$ & $293(2)$ & $293(2)$ & $293(2)$ & $293(2)$ & $293(2)$ \\
\hline $\mathrm{R} 1, \mathrm{wR} 2[\mathrm{I}>2 \sigma(\mathrm{I})]$ & $0.0548,0.1422$ & $0.0613,0.1462$ & $0.1460,0.3475$ & $0.1513,0.4055$ & $0.1285,0.3186$ \\
\hline R1,wR2 (all data) & $0.0894,0.1659$ & $0.1272,0.1741$ & $0.3012,0.4385$ & $0.2244,0.4439$ & $0.1722,0.3472$ \\
\hline Quality of fit & 1.066 & 1.007 & 1.025 & 1.236 & 1.127 \\
\hline
\end{tabular}

